\title{
Refinement of the Robert-Bonamy formalism: Considering effects from the line coupling
}

\author{
Q. Ma, ${ }^{1}$ C. Boulet, ${ }^{2}$ and R. H. Tipping ${ }^{3}$ \\ ${ }^{1}$ NASA/Goddard Institute for Space Studies and Department of Applied Physics and Applied Mathematics, \\ Columbia University, 2880 Broadway, New York, New York 10025, USA \\ ${ }^{2}$ Institut des Sciences Moléculaires d'Orsay (ISMO), CNRS (UMR8214) and Université Paris-Sud Bât 350, \\ Campus d'Orsay F-91405, France \\ ${ }^{3}$ Department of Physics and Astronomy, University of Alabama, Tuscaloosa, Alabama 35487-0324, USA
}

(Received 16 April 2013; accepted 24 June 2013; published online 17 July 2013)

\begin{abstract}
Since it was developed in 1979, the Robert-Bonamy (RB) formalism has been widely used in calculating pressure broadened half-widths and induced shifts for many molecular systems. However, this formalism contains several approximations whose applicability has not been thoroughly justified. One of them is that lines of interest are well isolated. When these authors developed the formalism, they have relied on this assumption twice. First, in calculating the spectral density $F(\omega)$, they have only considered the diagonal matrix elements of the relaxation operator. Due to this simplification, effects from the line mixing are ignored. Second, when they applied the linked cluster theorem to remove the cutoff, they have assumed the matrix elements of the operator $\exp \left(-\mathrm{i} \mathrm{S}_{1}-\mathrm{S}_{2}\right)$ can be replaced by the exponential of the matrix elements of $-i S_{1}-S_{2}$. With this replacement, effects from the line coupling are also ignored. Although both these two simplifications relied on the same approximation, their validity criteria are completely different and the latter is more stringent than the former. As a result, in many cases where the line mixing becomes negligible, significant effects from the line coupling have been completely missed. In the present study, we have developed a new method to evaluate the matrix elements of $\exp \left(-\mathrm{iS}_{1}-\mathrm{S}_{2}\right)$ and have refined the RB formalism such that line coupling can be taken into account. Our numerical calculations of the half-widths for Raman $\mathrm{Q}$ lines of the $\mathrm{N}_{2}-\mathrm{N}_{2}$ pair have demonstrated that effects from the line coupling are important. In comparison with values derived from the RB formalism, new calculated values for these lines are significantly reduced. A recent study has shown that in comparison with the measurements and the most accurate close coupling calculations, the RB formalism overestimates the half-widths by a large amount. As a result, the refinement of the RB formalism goes in the right direction and these new calculated half-widths become closer to the "true" values. () 2013 AIP Publishing LLC. [http://dx.doi.org/10.1063/1.4813234]
\end{abstract}

\section{INTRODUCTION}

Knowledge of molecular spectroscopy is essential for atmospheric sensing, combustion diagnostic, and other scientific and engineering applications. Of all spectroscopic line parameters, the accurate determination of pressure broadened half-width and induced shift is the most difficult, and remains as a challenge for experimentalists and theorists. With respect to theoretical efforts starting from several decades ago, many researchers have developed different lines shape theories categorizing in the purely classical method, ${ }^{1}$ the semi-classical method, ${ }^{2,3}$ and the fully quantum approach. ${ }^{4}$ At present, the close coupling method ${ }^{4}$ in the third category is the most accurate one. Mainly due to larger numbers of coupling quantum channels, the close coupling calculations are not feasible for many molecular systems except for those consisting of two linear molecules or even simpler ones. For more complicated systems, one has to rely on semi-classical and purely classical methods. Readers can find detailed explanations about these approaches in a book. ${ }^{5}$

The first semi-classical pressure broadening theory was proposed by Anderson ${ }^{2}$ in 1949 and later, it was more com- prehensively presented by Tsao and Curnutte $^{3}$ in 1962 . Their work is now commonly called as the ATC theory. Several main refinements of the ATC theory, such as a better trajectory description, a better perturbative treatment of the scattering operator, and a consideration of more realistic potential models containing short range components, have been proposed by many researchers in order to overcome its weaknesses. One can find a detailed analysis of these improvements in Ref. 6. Here, we only outline some of the major steps in the evolution process.

In 1970, Herman and Tipping introduced a more realistic description of the trajectory for close collisions. ${ }^{7}$ That model was later improved by Bonamy et al. by introducing an approximate parabolic trajectory model. ${ }^{8}$ In 1975, Leavitt and Korff showed that with an approximate re-summation of the infinite series of the scattering operator, how the linked cluster theorem ${ }^{9}$ for degenerate cases could lead to a bounded exponential form of the interruption function..$^{10}$ Later, a major progress was made by Robert and Bonamy in 1979 such that besides applying the linked cluster theorem to evaluate the Liouville scattering operator, these authors also considered short range interactions by introducing an atom-atom 
potential model. ${ }^{11}$ Meanwhile, they were still able to keep an analytical treatment of the interruption functions. These new features are the backbone of the well-known Robert-Bonamy (RB) formalism.

Then in 1992, Bykov et al. proposed a new way to improve trajectory models by introducing "exact" trajectories determined by isotropic potentials and the latter can be calculated with the classical mechanics. ${ }^{12}$ Later, their suggestion was realized by Buldyreva et al. ${ }^{13}$ Of course, the "exact" trajectory model is not really exact because in evaluating trajectories, effects from anisotropic interactions have been completely ignored. But, given the fact that all other models have suffered from this approximation and the "exact" trajectory model is more accurate than others, the "exact" model has become more common in recent years. Despite that it requires more CPU time, thanks to advance of computer power, the model has been adopted in most recent calculations.

More recently, we have shown that there is a subtle derivation error in developing the $\mathrm{RB}$ formalism. ${ }^{14}$ The error results from that with respect to the perturber states in Hilbert space, the Liouville scattering operator is not diagonal and the diagonalization itself is a basic requirement to apply the linked cluster theorem. We have proposed a modified version of the RB formalism based on a correct way to apply the cumulant expansion. ${ }^{15}$ The latter is equivalent to the linked cluster theorem, but its applications are more general. Furthermore by introducing the coordinate representation, we have completely overcome a longstanding problem of convergence commonly existing in most of theoretical calculations of the half-width and shift due to adopting more realistic potentials containing the short-range atom-atom model. ${ }^{16}$

Despite all efforts to improve the RB formalism such as adjusting the potential parameters, the current RB formalism can yield reasonably good agreement with measurements for some systems. However, a series of recent papers ${ }^{17,18}$ have demonstrated that for simpler systems where results of the close coupling calculations with more sophisticated potentials are available, the RB formalism significantly overestimates the half-widths. Because there is no room to make potential adjustments, the large differences clearly mean that the RB formalism itself contains some weaknesses. In fact, until now the RB formalism still contains several main assumptions whose applicability has not been thoroughly justified.

In the present study, we have scrutinized one of the major approximations, i.e., the isolated line approximation (or sometimes called as the well separated line approximation). We have found that in developing the RB formalism, Robert and Bonamy have relied on this approximation twice. First, in calculating the spectral density $\mathrm{F}(\omega)$, they have only considered the diagonal matrix elements of the relaxation operator. Due to this simplification, effects from the line mixing are ignored. Second, when they applied the linked cluster theorem to remove the cutoff appearing in the ATC theory, they have assumed matrix elements of an exponential function of the operator can be replaced by values of an ordinary exponential function whose arguments are the matrix elements of this operator. In other words, they have assumed that behavior of an exponential of the operator looks like an ordinary function. With this replacement, effects from the line coupling are ignored. It is worth mentioning that although both these two simplifications rely on the same approximation, their validity criteria are completely different and, as shown later, the latter is more stringent than the former. As a result, in many cases where the line mixing becomes negligible, effects on calculated half-widths and shifts from the line coupling remain important and they have been completely ignored.

The line mixing is a well known phenomenon. ${ }^{5}$ With the binary collision approximation valid in atmospheric environments, its fundamental theory has been established in terms of the relaxation operator in the line space of the absorber molecule. Real and imaginary parts of its diagonal matrix elements represent half-widths and shifts of the corresponding lines. Meanwhile, its off-diagonal elements cause line collisional interferences. For overlapping lines, no matter if the overlaps result from their original frequency positions or from high perturber pressures, the line mixing is one of the important factors that can vastly alter the line shape. ${ }^{5}$ Within the usual RB formalism, one cannot include the line mixing because the off-diagonal matrix elements of the relaxation operator have been assumed to be zero.

On the other hand, in modern line shape theories where the Liouville scattering operator is given in terms of the exponential form, whether the latter results from the cumulant expansion or some other ways, the line coupling does exist. This subject has been studied by several researchers. Among them, Cherkasov has pursued this study for more than three decades. ${ }^{19}$ In developing his formalism, some practical difficulties have forced him to introduce an additional approximation. In the present study, we have shown that with the modified RB formalism where the cumulant expansion is correctly applied, one is able to avoid these difficulties. Without introducing his approximation and without adding a restriction to limit the number of coupled lines to be included, one is able to correctly and effectively consider the line coupling in practical calculations.

In Sec. II, we present how the refinement of the RB formalism is built in detail. In Sec. III, we apply the refined formalism to the $\mathrm{N}_{2}-\mathrm{N}_{2}$ system and present calculated halfwidths of the Raman $\mathrm{Q}$ lines. Based on these numerical results, we explicitly demonstrate how important the effects on calculated half-widths from the line coupling are. In Sec. IV, we present discussions and conclusions.

\section{THEORY}

\section{A. General formalism of the absorption coefficient and the spectral density}

It is well known that the absorption coefficient for radiation caused by a gaseous sample with $\mathrm{n}_{\mathrm{a}}$ absorber molecules per unit volume in thermal equilibrium at temperature $\mathrm{T}$ is related to the spectral density $F(\omega)$

$$
\alpha(\omega)=\frac{4 \pi^{2}}{3 \hbar c} n_{a} \omega\left(1-e^{-\beta \hbar \omega}\right) F(\omega) .
$$


Meanwhile, $F(\omega)$ is defined by

$$
F(\omega)=-\frac{1}{\pi} \operatorname{Im} \operatorname{Tr}_{a}\left\{X^{\dagger} \frac{1}{\omega-\mathcal{L}_{a}-T r_{b a t h}\left\{M(\omega) \rho_{b}\right\}} \rho_{a} X\right\},
$$

where $\mathrm{X}$ is the transition operator. With the binary collision approximation valid for atmospheric pressures and the impact approximation applicable for a frequency region around the line centers, $\operatorname{Tr}_{\text {bath }}\left\{\mathbf{M}(\omega) \rho_{\mathrm{b}}\right\}$ can be given by ${ }^{20}$

$$
\operatorname{Tr}_{b a t h}\left\{M(\omega) \rho_{b}\right\}=n_{b}\langle m(0)\rangle_{b a t h},
$$

where $n_{b}$ is the number density of the bath molecule, $m(0)$ is a binary-collision operator, and $\langle\cdots\rangle_{\text {bath }}$ denotes averages over the internal degrees of the bath molecule as well as averages over the translational degrees of the interacting pair. Usually, $\langle\mathrm{m}(0)\rangle_{\text {bath }}$ is called the relaxation operator. Then, by following Ben-Reuven's convention ${ }^{21}$ and introducing $\mid$ if,$\left.\left.J M_{J}\right\rangle\right\rangle$ defined by

$$
\begin{aligned}
\left.\left|i f, J M_{J}\right\rangle\right\rangle= & \sum_{\mathrm{m}_{\mathrm{i}} \mathrm{m}_{\mathrm{f}}}(-1)^{j_{f}-m_{f}} \\
& \times C\left(j_{i} j_{f} J, m_{i}-m_{f} M_{J}\right)\left|i m_{i} f m_{f}\right\rangle,
\end{aligned}
$$

the spectral density $\mathrm{F}(\omega)$ can be expressed as

$$
\begin{aligned}
F(\omega)= & -\frac{1}{(2 J+1) \pi} \operatorname{Im} \sum_{i f} \sum_{i^{\prime} f^{\prime}} X_{i^{\prime} f^{\prime}}^{\Pi J *} X_{i f}^{\Pi J} \rho_{i} \\
& \times\left\langle\left\langle i^{\prime} f^{\prime}, J M_{J}\left|\frac{1}{\omega-\mathcal{L}_{a}-n_{b}\langle m(0)\rangle_{\text {bath }}}\right| i f, J M_{J}\right\rangle\right\rangle .
\end{aligned}
$$

In the above expression, $X_{i f}^{\Pi J}$ is a simple notation for $\sqrt{2 j_{i}+1}\left\langle i\left\|X^{\Pi J}\right\| f\right\rangle$ where $\Pi$ indicates the parity of $\mathrm{X}^{21}$ and the reduced matrix elements $\left\langle i\left\|X^{\Pi J}\right\| f\right\rangle$ result from

$$
\begin{aligned}
& \left\langle i m_{i}\left|X_{M_{J}}^{\Pi J}\right| f m_{f}\right\rangle \\
& =(-1)^{j_{f}-m_{f}} C\left(j_{i} j_{f} J, m_{i}-m_{f} M_{J}\right) \sqrt{\frac{2 j_{i}+1}{2 J+1}}\left\langle i\left\|X^{\Pi J}\right\| f\right\rangle .
\end{aligned}
$$

In 1979, based on the linked cluster theorem, ${ }^{9}$ Robert and Bonamy had developed the RB formalism characterized by a non-perturbative treatment of the Liouville scattering operator $\hat{\mathrm{S}} .{ }^{11}$ Despite its popularity, ${ }^{5,6}$ the RB formalism itself suffers from severe weaknesses resulting from unjustified approximations. One of them is the assumption that lines of interest are isolated. As mentioned above, they have relied on this assumption twice. One occurs at this stage: they have only considered the diagonal matrix elements of $n_{b}\langle m(0)\rangle_{\text {bath }}$ in Eq. (5). The validity criterion of this simplification is matrix elements of $n_{b}\langle m(0)\rangle_{\text {bath }}$ are significantly smaller than values of the diagonal operator $\omega-\mathcal{L}_{a}$. In general, this criterion is not too stringent and it is valid for many cases unless lines positions become too close and/or the number density $\mathrm{n}_{\mathrm{b}}$ is too high. In fact, as long as gaps between lines of interest are much larger than their half-widths, one can consider the criterion is valid and thus, at least in the intense absorption regions near line centers, one can ignore effects from the line mixing in calculations. However, we would like to mention that in troughs in between transition lines and in far wings of vibrational bands, the line mixing could strongly affect absorptions even if gaps between lines are larger than their widths. ${ }^{5}$ In the present study, we mainly focus our attention to situations where the line mixing can be neglected. Then, $F(\omega)$ can be simplified as

$$
\begin{aligned}
F(\omega)= & -\frac{1}{(2 J+1) \pi} \operatorname{Im} \sum_{i f}\left|X_{i f}^{\Pi J}\right|^{2} \rho_{i} \\
& \times \frac{1}{\omega-\omega_{i f}-n_{b}\left\langle\left\langle i f, J M_{J}\left|\langle m(0)\rangle_{\text {bath }}\right| i f, J M_{J}\right\rangle\right\rangle} .
\end{aligned}
$$

\section{B. Different ways in applying the cumulant expansion and their consequences}

In Fano's notation, the relaxation operator $\langle\mathrm{m}(0)\rangle_{\text {bath }}$ is related to the Liouville scattering operator $\hat{\mathrm{S}}\left(=\mathrm{S}_{\mathrm{I}} \mathrm{S}_{\mathrm{F}}^{*}\right)$ by

$$
\langle m(0)\rangle_{b a t h}=\frac{i}{2 \pi}\langle 1-\hat{S}\rangle_{b a t h} .
$$

How to evaluate the matrix elements of $\hat{S}$ is a crucial step in developing line shape theories. It is well known that the cumulant expansion ${ }^{15}$ is a useful tool and has been widely applied in many physical problems. A key problem in applying this method is to define an average usually denoted by \langle\rangle . Its accepted definitions could be very general such as diagonal matrix elements or something else. But the average must satisfy the normalization condition $\langle\mathrm{I}\rangle=1$ where $\mathrm{I}$ is an identity operator. As shown in Appendix A, by applying the cumulant expansion for the Liouville operator $\hat{S}$, its average up to the second order can be written as

$\langle\hat{S}\rangle=\left\langle\theta \exp \left(-\frac{i}{\hbar} \int_{-\infty}^{\infty} \mathcal{L}_{1}(t) d t\right)\right\rangle=\exp \left(-i S_{1}-S_{2}\right)$,

where $S_{1}$ and $S_{2}$ are defined by Eqs. (A5) and (A6), respectively.

Several years ago, by scrutinizing Robert and Bonamy's derivations, we have found that there is a subtle error in their applying the linked cluster theorem. ${ }^{14}$ Because the core part of the linked cluster theorem is equivalent to the cumulant expansion, we prefer to present our discussions in terms of the cumulant expansion. In order to remedy their mistake, we have developed a correct way to apply the cumulant expansion. The essential difference between these two applications results from two different choices of the average required in applying the cumulant expansion. With our choice, the average is defined as an average over the whole internal degrees in the bath molecular line space. As a result, the operators of $S_{1}$ and $S_{2}$ appearing in the exponential function are independent of states of the bath molecule. In contrast, the average defined by them only covers a part of the internal degrees in the bath molecular line space and thus, $S_{1}$ and $S_{2}$ depend on the both molecular states. For clarity purpose, we distinguish these operator symbols by adding the subscripts of $\mathrm{RB}$ or modRB 
accordingly. Then, one can describe their relations as

$$
S_{1 o r 2, \bmod R B}=\left\langle S_{1 o r 2, R B}\right\rangle_{i_{2}} \equiv \sum_{i_{2}}\left(2 i_{2}+1\right) \rho_{i_{2}} S_{1 o r 2, R B} .
$$

In other words, $S_{1 \text { or2,mod RB }}$ are averages of $S_{1 \text { or2,RB }}$ over states of the bath molecule.

There are significant consequences from this intrinsic difference. First of all, by starting from the correct choice of the average but exactly following the same further steps as Robert and Bonamy did, we have obtained different formulas for the half-width and the shift. ${ }^{14}$ Within the RB formalism, the halfwidth $\gamma_{\mathrm{RB}}$ is given by

$$
\begin{aligned}
\gamma_{R B}= & \frac{n_{b}}{2 \pi c} \int_{0}^{+\infty} v f(v) d v \int_{0}^{+\infty} 2 \pi b d b \\
& \times\left\langle 1-\cos \left[S_{1}+\operatorname{Im}\left(S_{2}\right)\right] e^{-\operatorname{Re}\left(S_{2}\right)}\right\rangle_{i_{2}},
\end{aligned}
$$

where $\mathrm{f}(\mathrm{v})$ is the Maxwell-Boltzmann distribution function. On the other hand, within the modified RB formalism, the half-width $\gamma_{\bmod R B}$ is given by

$$
\begin{aligned}
\gamma_{\bmod R B}= & \frac{n_{b}}{2 \pi c} \int_{0}^{+\infty} v f(v) d v \int_{0}^{+\infty} 2 \pi b d b \\
& \times\left\{1-\cos \left[\left\langle S_{1}\right\rangle_{i_{2}}+\operatorname{Im}\left(\left\langle S_{2}\right\rangle_{i_{2}}\right)\right] e^{-\operatorname{Re}\left(\left\langle S_{2}\right\rangle_{i_{2}}\right)}\right\} .
\end{aligned}
$$

Readers can find the formulas for the shift derived from the original RB formalism and that from the modified RB formalism in our work. ${ }^{14}$ Unfortunately, when we made this modification, we did not scrutinize their approach in evaluating $\exp \left(-\mathrm{iS}_{1}-\mathrm{S}_{2}\right)$ and simply adopted their assumption that one needs only to consider diagonal matrix elements for any operators involved. As a result, without making full justification, effects from the line coupling are completely ignored in both Eqs. (11) and (12).

Recently, we have realized when one tries to accurately evaluate the matrix of $\exp \left(-i \mathrm{~S}_{1}-\mathrm{S}_{2}\right)$, there is a more profound consequence occurring. Because the operator $\left(-i S_{1}-S_{2}\right)$ is off-diagonal in its original representation, a proper way to evaluate matrix elements of $\exp \left(-\mathrm{i} \mathrm{S}_{1}-\mathrm{S}_{2}\right)$ is to diagonalize $\left(-\mathrm{iS}_{1}-\mathrm{S}_{2}\right)$ first. Then, by choosing a new representation constructed by a set of its eigenvectors, the matrix of $\left(-\mathrm{S}_{1}-\mathrm{S}_{2}\right)$ becomes diagonal. The advantage of being a diagonal matrix is that the matrix of $\exp \left(-\mathrm{iS}_{1}-\mathrm{S}_{2}\right)$ is also diagonal. The latter's diagonal elements are values of the exponential function whose arguments are just values of the diagonal elements of $\left(-i S_{1}-S_{2}\right)$. Finally, by projecting the eigenvectors into the original basis to carry out unitary transformations between these two representation, one can obtain exact matrix element values of $\exp \left(-\mathrm{i} \mathrm{S}_{1}-\mathrm{S}_{2}\right)$ without any distortions.

Within the RB formalism, the size of $\left(-i S_{1}-S_{2}\right)$ is determined by a product of the number of coupled absorber's lines and the number of bath molecular states labeled by $i_{2}$. As a result, the size of matrices required to diagonalize would be very large. Consequently, both the number of the eigenvectors and the latter's dimensions become very large too.
Besides, as shown in Eq. (11), in calculating the half-width with the RB formalism, an additional procedure to perform an average over the bath states $i_{2}$ is required. During this procedure, one has to repeatedly project the eigenvectors into the corresponding $i_{2}$ axes. All these practices contain heavy computational burdens. Furthermore, the operator of $\left(-i \mathrm{~S}_{1}-\mathrm{S}_{2}\right)$ depends on the impact parameter $b$ (or the closest distance $r_{c}$ ) used to specify trajectories. This implies that during a course to perform an average over the translational motion, the diagonalization and the projection procedures have to be carried out many times. As a result, to consider the line coupling is not feasible in practice.

On the other hand, within the modified RB formalism, the size of $-i S_{1}-S_{2}$ becomes much smaller because it is only determined by the number of coupled lines. In addition, any procedures involving the bath molecule are completely obviated. Thus, with reasonable computational efforts, one is able to effectively and accurately consider effects from the line coupling. In summary, it is the correct choice in applying the cumulant expansion that allows us to overcome one of longstanding weaknesses of the RB formalism.

As an example, we consider the Raman Q lines of the $\mathrm{N}_{2}-\mathrm{N}_{2}$ pair. In carrying out the average over $\mathrm{i}_{2}$, if one takes into account the states up to $i_{2 \max }=40$, the size of the matrices required to diagonalize in the RB formalism would be 41 times larger than that in the modified RB formalism. (In our numerical calculations presented later, we have chosen $\mathrm{i}_{2 \max }$ $=60$.) Meanwhile, thanks to the fact that even $Q(j)$ lines do not coupled with odd $Q(j)$ lines, the number of coupled lines is reduced by a half. Then, if one considers the line coupling among the even $\mathrm{Q}(\mathrm{j})$ lines up to $\mathrm{j}_{\max }=30$, the sizes of matrices in the RB and modified RB formalisms are $656 \times 656$ and $16 \times 16$, respectively. It is well known that numerical burden to diagonalize a $\mathrm{M} \times \mathrm{M}$ matrix increases very quickly as $\mathrm{M}^{3}$ increases. Then, roughly speaking, in comparison with the RB formalism, the computational burden required with the modified RB formalism is reduced by 68900 times.

The original purpose to introduce the cumulant expansion was to remove the cut-off appearing in the ATC theory. However, the cumulant expansion brings other benefits as well. One of them is to provide a room to take into account the line coupling. But, it is the correct way in applying the cumulant expansion that enables one to exploit this benefit.

\section{Expression for the half-width with including the line coupling}

After correctly applying the cumulant expansion, one needs to accurately evaluate the matrix elements of $\left\langle\left\langle\mathrm{i}^{\prime} \mathrm{f}^{\prime}\right| \exp \left(-\mathrm{i} \mathrm{S}_{1}-\mathrm{S}_{2}\right)\right.$ lif $\rangle$. At this stage, the first task is to construct the whole matrix of $-i S_{1}-S_{2}$ in the line space. The job requires not only evaluations of its diagonal matrix elements but also its off-diagonal elements as well. Expressions for the former are available, but one needs to derive new expressions for the latter. In this case, the size of this matrix is determined by number of lines which are coupled to each other through the operator $-\mathrm{iS}_{1}-\mathrm{S}_{2}$. Symmetry considerations enable one to reduce the size. In addition, one 
can drop very weakly coupled lines from consideration. In general, we expect that sizes of matrices would be reasonable, no more than several dozens. The next task is by diagonalizing the matrix constructed, to find its eigenvalues and eigenvectors.

Let us assume that the eigenvalues and eigenvectors of $-\mathrm{i} \mathrm{S}_{1}-\mathrm{S}_{2}$ are denoted by $\mathrm{G}\left(\eta_{\mathrm{n}}\right)$ and $\left.\left|\eta_{\mathrm{n}}\right\rangle\right\rangle$ with $\mathrm{n}=1, \ldots, \mathrm{N}$ where $\mathrm{N}$ is the number of the coupled lines. Then, in terms of $\mathrm{G}\left(\eta_{\mathrm{n}}\right)$ and $\left.\left|\eta_{\mathrm{n}}\right\rangle\right\rangle,\left\langle\left\langle\mathrm{i}^{\prime} \mathrm{f}^{\prime}\right| \exp \left(-\mathrm{i} \mathrm{S}_{1}-\mathrm{S}_{2}\right)\right.$ lif $\left.\rangle\right\rangle$ are given by

$$
\left\langle\left\langle i^{\prime} f^{\prime}\left|e^{-i S_{1}-S_{2}}\right| i f\right\rangle\right\rangle=\sum_{n}\left\langle\left\langle i^{\prime} f^{\prime} \mid \eta_{n}\right\rangle\right\rangle e^{G\left(\eta_{n}\right)}\left\langle\left\langle\eta_{n} \mid i f\right\rangle\right\rangle .
$$

In general, the operator of $-\mathrm{iS}_{1}-\mathrm{S}_{2}$ is not Hermitian. As a result, $\mathrm{G}\left(\eta_{\mathrm{n}}\right)$ are complex consisting of real and imaginary parts $\operatorname{ReG}\left(\eta_{\mathrm{n}}\right)$ and $\operatorname{ImG}\left(\eta_{\mathrm{n}}\right)$. Similarly, $\left\langle\left\langle\mathrm{i}^{\prime} \mathrm{f}^{\prime} \mid \eta_{\mathrm{n}}\right\rangle\right.$ and $\left\langle\left\langle\eta_{\mathrm{n}}\right|\right.$ if $\left.\rangle\right\rangle$ are complex too. For the diagonal matrix elements of $\left\langle\left\langle\right.\right.$ if $\left|\exp \left(-\mathrm{iS}_{1}-\mathrm{S}_{2}\right)\right|$ if $\left.\rangle\right\rangle$, one can express their real part as

$$
\operatorname{Re}\left\langle\left\langle i f\left|e^{-i S_{1}-S_{2}}\right| i f\right\rangle\right\rangle=\sum_{n}\left|\left\langle\left\langle i f \mid \eta_{n}\right\rangle\right\rangle\right|^{2} \cos \left[\operatorname{Im} G\left(\eta_{n}\right)\right] e^{\operatorname{Re} G\left(\eta_{n}\right)} .
$$

The expression for their imaginary part is not presented here. Besides, it is easy to write down expressions for the offdiagonal matrix elements $\left\langle\left\langle\mathrm{i}^{\prime} \mathrm{f}^{\prime}\left|-\mathrm{i} \mathrm{S}_{1}-\mathrm{S}_{2}\right| \mathrm{if}\right\rangle\right\rangle$ which are also not presented here. Then, by carrying out averages over the translational motion for the factor of $\left\{1-\left\langle\langle\right.\right.$ if $| \exp \left(-i S_{1}-S_{2}\right)$ $\mid$ if $\rangle\rangle\}$, the refined expression for the half-width is given by

$$
\begin{aligned}
\gamma_{r e f}= & \frac{n_{b}}{2 \pi c} \int_{0}^{+\infty} v f(v) d v \int_{0}^{+\infty} 2 \pi b d b \\
& \times\left\{1-\sum_{n}\left|\left\langle\left\langle i f \mid \eta_{n}\right\rangle\right\rangle\right|^{2} \cos \left[\operatorname{Im} G\left(\eta_{n}\right)\right] e^{\operatorname{Re} G\left(\eta_{n}\right)}\right\} .
\end{aligned}
$$

In the above expression, effects on the half-widths from the line coupling are well included. Our numerical calculations will demonstrate the necessity of adopting these new formulas. The expression for the shift is similar to Eq. (15), but is not presented here.

\section{NUMERICAL CALCULATIONS FOR RAMAN Q LINES OF THE $\mathrm{N}_{2}-\mathrm{N}_{2}$ PAIR}

As a sample to show effects from the line coupling, we consider a system consisting of two linear molecules. Recently, Thibault et al. have reported their study on comparison of quantum, semi-classical and classical methods in calculating half-widths of the Raman $Q$ lines for the $\mathrm{N}_{2}-\mathrm{N}_{2}$ pair. ${ }^{18}$ They have found that in comparisons with measurements and values obtained from the close coupling calculations, results derived from the RB formalism and also the modified RB formalism (excluding the line coupling) are overestimated by a large amount. Because the potential model used by their calculations is available, ${ }^{22}$ we can calculate the half-widths from Eqs. (12) and (15). Then, by comparing our results with the close coupling ones (in very good agreement with experimental data), we will be able to quantitatively access effects from the line coupling. We would like to note that the potential energy surface used here and in Ref. 18 does not contain vibra- tional dependences. Consequently, vibrational effects are excluded in these calculations. However, the previous studies ${ }^{18}$ have shown that to include the vibrational dependence has a little influence on calculated results.

\section{A. General expression for matrix elements of a Liouville operator after applying the cumulant expansion}

Because consideration of the line coupling in the modified RB formalism is a new subject, we need to derive some new formulas. We first introduce the unit vector $\left.\left|\mathrm{I}_{\mathrm{b}}\right\rangle\right\rangle$ and the density vector $\left.\left|\rho_{\mathrm{b}}\right\rangle\right\rangle$ in the line space of the bath molecule defined by

$$
\begin{aligned}
& \left.\left.\left|I_{b}\right\rangle\right\rangle=\sum_{i_{2} m_{2}}\left|i_{2} m_{2} i_{2} m_{2}\right\rangle\right\rangle, \\
& \left.\left.\left|\rho_{b}\right\rangle\right\rangle=\sum_{i_{2} m_{2}} \rho_{i_{2}}\left|i_{2} m_{2} i_{2} m_{2}\right\rangle\right\rangle .
\end{aligned}
$$

In terms of these two vectors, the average \langle\rangle defined in applying the cumulant expansion can be explicitly expressed as

$$
\langle\hat{O}\rangle=\left\langle\left\langle I_{b}|\hat{O}| \rho_{b}\right\rangle\right\rangle,
$$

where $\hat{O}$ is a Liouville operator of interest. It is obvious that the normalization condition $\left\langle\hat{I}_{b}\right\rangle=1$ is satisfied. Then in terms of the cumulant expansion, matrix elements of $\langle\hat{\mathrm{O}}\rangle_{\text {bath }}$ in the line space of the absorber molecule can be expressed as

$$
\begin{aligned}
& \left\langle\left\langle i^{\prime} f^{\prime}, J M_{J}\left|\langle\hat{O}\rangle_{\text {bath }}\right| i f, J M_{f}\right\rangle\right\rangle \\
& \quad=T r_{\text {trans }}\left\{\left\langle\left\langle i^{\prime} f^{\prime}, J M_{J} ; I_{b}|\hat{O}| i f, J M_{J} ; \rho_{b}\right\rangle\right\rangle\right\},
\end{aligned}
$$

where

$$
\left.\left.\left.\left.\left.\mid i f, J M_{J} ; I_{b} \text { or } \rho_{b}\right\rangle\right\rangle=\left|i f, J M_{J}\right\rangle\right\rangle \otimes \mid I_{b} \text { or } \rho_{b}\right\rangle\right\rangle .
$$

In Eq. (18), $\operatorname{Tr}_{\text {trans }}\{\}$ means the average over the translational motion. Finally, in order to fully exploit the rotation invariance, ${ }^{21}$ one prefers to replace these matrix elements by their averages over the quantum number $\mathrm{M}_{\mathrm{J}}$. It implies one makes a replacement such that

$$
\begin{aligned}
& \left\langle\left\langle i^{\prime} f^{\prime}, J M_{J} ; I_{b}|\hat{O}| i f, J M_{J} ; \rho_{b}\right\rangle\right\rangle \\
& \quad=\frac{1}{2 J+1} \sum_{M_{J}}\left\langle\left\langle i^{\prime} f^{\prime}, J M_{J} ; I_{b}|\hat{O}| i f, J M_{J} ; \rho_{b}\right\rangle\right\rangle .
\end{aligned}
$$

Eq. (20) is the starting point in the following derivations.

\section{B. Matrix elements of $\left\langle L_{1}(\mathrm{t})\right\rangle,\left\langle L_{1}(\mathrm{t}) L_{1}\left(\mathrm{t}^{\prime}\right)\right\rangle$, and $\left\langle L_{1}(\mathrm{t})\right\rangle\left\langle L_{1}\left(\mathrm{t}^{\prime}\right)\right\rangle$ in the line space}

In practice, by considering up to the second order of the cumulant expansion, expressions for $S_{1}$ and $S_{2}$ are required. As shown in Eqs. (A5) and (A6), one needs to explicitly know what these $\left\langle L_{1}(\mathrm{t})\right\rangle,\left\langle L_{1}(\mathrm{t}) L_{1}\left(\mathrm{t}^{\prime}\right)\right\rangle$, and $\left\langle L_{1}(\mathrm{t})\right\rangle\left\langle L_{1}\left(\mathrm{t}^{\prime}\right)\right\rangle$ terms are first. With the choice of the average defined by Eq. (17), these terms are Liouville operators in the line space of the absorber molecule and contain a classical parameter used to specify the trajectory. With Eqs. (4), (16), and (20), an explicit expression 
for the matrix elements of $\left\langle L_{1}(\mathrm{t})\right\rangle$ can be written as

$$
\begin{aligned}
\left\langle\left\langle i^{\prime} f^{\prime},\right.\right. & \left.\left.J M_{J}\left|\left\langle\mathcal{L}_{1}(t)\right\rangle\right| i f, J M_{J}\right\rangle\right\rangle \\
= & \left.\left\langle i^{\prime} f^{\prime}, J M_{J} ; I_{b}\left|\mathcal{L}_{1}(t)\right| i f, J M_{J} ; \rho_{b}\right\rangle\right\rangle \\
= & \frac{1}{2 J+1} \sum_{i_{2} m_{2}} \rho_{i_{2}} \sum_{i_{2}^{\prime} m_{2}^{\prime}} \sum_{(m)}(-1)^{j_{f}-m_{f}+j_{f}^{\prime}-m_{f}^{\prime}} \\
& \times C\left(j_{i}^{\prime} j_{f}^{\prime} J, m_{i}^{\prime}-m_{f}^{\prime} M_{J}\right) C\left(j_{i} j_{f} J, m_{i}-m_{f} M_{J}\right) \\
& \times\left\langle\left\langle i^{\prime} m_{i}^{\prime} f^{\prime} m_{f}^{\prime}, i_{2}^{\prime} m_{2}^{\prime} i_{2}^{\prime} m_{2}^{\prime}\left|\mathcal{L}_{1}(t)\right| i m_{i} f m_{f}, i_{2} m_{2} i_{2} m_{2}\right\rangle,\right.
\end{aligned}
$$

where a summary notation $(\mathrm{m})$ means summations over all magnetic quantum numbers associated with the absorber molecule and a summation over $\mathrm{M}_{\mathrm{J}}$ as well. In terms of matrix elements in Hilbert space, the expression for the matrix elements of $L_{1}(\mathrm{t})$ is presented in Appendix B. With Eq. (B1), one can rewrite Eq. (21) as

$$
\begin{aligned}
& \left\langle\left\langle i^{\prime} f^{\prime}, J M_{J}\left|\left\langle\mathcal{L}_{1}(t)\right\rangle\right| i f, J M_{J}\right\rangle\right\rangle \\
& =\sum_{i_{2}}\left(2 i_{2}+1\right) \rho_{i_{2}}\left\{\left\langle i^{\prime} i_{2}\left|V_{i s o}(t)\right| i i_{2}\right\rangle \delta_{j_{i}^{\prime} j_{i}} \delta_{f^{\prime} f}\right. \\
& \left.\quad-\left\langle f i_{2}\left|V_{i s o}(t)\right| f^{\prime} i_{2}\right\rangle \delta_{j_{f} j_{f}^{\prime}} \delta_{i^{\prime} i}\right\} .
\end{aligned}
$$

We note that in Eq. (22), the Kronecker delta $\delta_{j_{i}^{\prime} j_{i}}$ means the angular moment $\mathrm{j}_{\mathrm{i}}^{\prime}$ must equal to the angular moment $\mathrm{j}_{\mathrm{i}}$, meanwhile the Kronecker delta $\delta_{i^{\prime} i}$ means all the quantum numbers associated with the state of $i^{\prime}$ must equal to their corresponding partners associated with the state of i. Based on Eq. (22), one obtains the expressions for the matrix elements of $S_{1}$ as

$$
\begin{aligned}
& \left\langle\left\langle i^{\prime} f^{\prime}, J M_{J}\left|S_{1}\right| i f, J M_{J}\right\rangle\right\rangle \\
& =\sum_{i_{2}}\left(2 i_{2}+1\right) \rho_{i_{2}}\left\{\delta_{j_{i}^{\prime} j_{i}} \delta_{f^{\prime} f} \frac{1}{\hbar} \int_{-\infty}^{\infty} d t\left\langle i^{\prime} i_{2}\left|V_{i s o}(t)\right| i i_{2}\right\rangle\right. \\
& \left.\quad-\delta_{j_{f} j_{f}^{\prime}} \delta_{i^{\prime} i} \frac{1}{\hbar} \int_{-\infty}^{\infty} d t\left\langle f i_{2}\left|V_{i s o}(t)\right| f^{\prime} i_{2}\right\rangle\right\} .
\end{aligned}
$$

With Eq. (23), one can conclude that only the isotropic part of potentials makes contributions to them and in cases that lines of interest belong to the same vibrational bands, these matrix elements are always diagonal. Because the potential model used in the present study does not depend on the vibrational quantum numbers, the $S_{1}$ term disappears.

Similarly, with respect to matrix elements associated with $\left\langle L_{1}(\mathrm{t}) L_{1}\left(\mathrm{t}^{\prime}\right)\right\rangle$, one has

$$
\begin{aligned}
& \left\langle\left\langle i^{\prime} f^{\prime}, J M_{J}\left|\left\langle\mathcal{L}_{1}(t) \mathcal{L}_{1}\left(t^{\prime}\right)\right\rangle\right| i f, J M_{J}\right\rangle\right\rangle \\
& =\frac{1}{2 J+1} \sum_{i_{2} m_{2}} \rho_{i_{2}} \sum_{i_{2}^{\prime} m_{2}^{\prime}} \sum_{(m)}(-1)^{j_{f}-m_{f}+j_{f}^{\prime}-m_{f}^{\prime}} \\
& \quad \times C\left(j_{i}^{\prime} j_{f}^{\prime} J, m_{i}^{\prime}-m_{f}^{\prime} M_{J}\right) C\left(j_{i} j_{f} J, m_{i}-m_{f} M_{J}\right) \\
& \quad \times\left\langle\left\langle i^{\prime} m_{i}^{\prime} f^{\prime} m_{f}^{\prime}, i_{2}^{\prime} m_{2}^{\prime} i_{2}^{\prime} m_{2}^{\prime}\left|\mathcal{L}_{1}(t) \mathcal{L}_{1}\left(t^{\prime}\right)\right| i m_{i} f m_{f}, i_{2} m_{2} i_{2} m_{2}\right\rangle\right\rangle .
\end{aligned}
$$

In Eq. (24), the matrix elements of $L_{1}(\mathrm{t}) L_{1}\left(\mathrm{t}^{\prime}\right)$ in the line space become more complicated. Their derivations are presented in Appendix B and their expressions are given by Eq. (B3). As shown in Eq. (B3), these matrix elements consist of four terms. The first two terms solely depend on the final states or the initial states of lines, respectively. Meanwhile, the other two terms depend both on the final and initial states. Because derivations of the expression are complicated, we prefer to present the deriviations separately for these terms. In the literature, people usually label the first two terms by the subscripts "outer,i" and "outer,f," respectively, and label a combination of the last two terms by the subscript of "middle." As an example, we consider the first term labeled as $\left\langle\mathbf{i}^{\prime} \mathrm{f}^{\prime}, \mathrm{JM}_{\mathbf{J}}\right.$ $\left|\left\langle L_{1}(\mathrm{t}) L_{1}\left(\mathrm{t}^{\prime}\right)\right\rangle\right|$ if, JM $\left.\left.\mathrm{J}_{\mathrm{J}}\right\rangle\right\rangle_{\text {outer,i. }}$. With Eqs. (24) and (B3), we have

$$
\begin{aligned}
& \left\langle\left\langle i^{\prime} f^{\prime}, J M_{J}\left|\left\langle\mathcal{L}_{1}(t) \mathcal{L}_{1}\left(t^{\prime}\right)\right\rangle\right| i f, J M_{J}\right\rangle\right\rangle_{\text {outer }, i} \\
& =\frac{\delta_{j_{i}^{\prime} j_{i}} \delta_{f^{\prime} f}}{2 j_{i}+1} \sum_{i_{2} m_{2}} \rho_{i_{2}} \sum_{m_{i}}\left\langle i^{\prime} m_{i} i_{2} m_{2}\left|\hat{V}(t) \hat{V}\left(t^{\prime}\right)\right| i m_{i} i_{2} m_{2}\right\rangle .
\end{aligned}
$$

With Eq. (25), one can conclude that this term would be diagonal as long as their initial states of lines of interest belong to the same vibrational bands. In other words, it only causes the line mixing between lines whose initial states belong to different bands. With respect to the second term $\left\langle i^{\prime} \mathrm{f}^{\prime}, \mathrm{JM}_{\mathrm{J}}\right.$ $\left|\left\langle L_{1}(\mathrm{t}) L_{1}\left(\mathrm{t}^{\prime}\right)\right\rangle\right|$ if,, $\left.\left.\mathrm{JM}_{\mathrm{J}}\right\rangle\right\rangle_{\text {outer, } \mathrm{f}}$, its expression is the same as Eq. (25), except for exchanges between $i$ and $\mathrm{f}^{\prime}$, between $\mathrm{i}^{\prime}$ and $\mathrm{f}$, and between $\mathrm{t}$ and $\mathrm{t}^{\prime}$. Meanwhile, one can conclude that this term only causes the line mixing between lines whose final states belong to different bands.

Concerning the combination term labeled by middle, we have

$$
\begin{aligned}
\left\langle\left\langle i^{\prime} f^{\prime},\right.\right. & \left.\left.J M_{J}\left|\left\langle\mathcal{L}_{1}(t) \mathcal{L}_{1}\left(t^{\prime}\right)\right\rangle\right| i f, J M_{J}\right\rangle\right\rangle_{m i d d l e} \\
= & -\frac{1}{2 J+1} \sum_{i_{2} m_{2}} \rho_{i_{2}} \sum_{i_{2}^{\prime} m_{2}^{\prime}} \sum_{(m)}(-1)^{j_{f}-m_{f}+j_{f}^{\prime}-m_{f}^{\prime}} \\
& \times C\left(j_{i}^{\prime} j_{f}^{\prime} J, m_{i}^{\prime}-m_{f}^{\prime} M_{J}\right) C\left(j_{i} j_{f} J, m_{i}-m_{f} M_{J}\right) \\
& \times\left\{\left\langle i^{\prime} m_{i}^{\prime} i_{2}^{\prime} m_{2}^{\prime}|\hat{V}(t)| i m_{i} i_{2} m_{2}\right\rangle\left\langle f m_{f} i_{2} m_{2}\left|\hat{V}\left(t^{\prime}\right)\right| f^{\prime} m_{f}^{\prime} i_{2}^{\prime} m_{2}^{\prime}\right\rangle\right. \\
& \left.+\left(t \leftrightarrow t^{\prime}\right)\right\} .
\end{aligned}
$$

In contrast with the first two terms which are diagonal for lines within the same bands, this term is always off-diagonal. This implies that no matter if lines of interest belong to the same or to different bands, this term can always cause the line coupling.

Finally, we consider the matrix elements of $\left\langle i^{\prime} \mathrm{f}^{\prime}, \mathrm{JM}_{\mathrm{J}}\right.$ $\left|\left\langle L_{1}(\mathrm{t})\right\rangle\left\langle L_{1}\left(\mathrm{t}^{\prime}\right)\right\rangle\right|$ if, JM $\left.\left.\mathrm{J}\right\rangle\right\rangle$. It has been known for years that only isotropic potentials make contributions to these matrix elements. In addition, these contributions exactly cancel out contributions to the matrix elements of $\left\langle\left\langle i^{\prime} \mathrm{f}^{\prime}, \mathrm{JM}_{\mathrm{J}}\left|\left\langle L_{1}(\mathrm{t}) L_{1}\left(\mathrm{t}^{\prime}\right)\right\rangle\right|\right.\right.$ if,, $\left.\left.\mathrm{JM}_{\mathrm{J}}\right\rangle\right\rangle$ from the isotropic potentials. As a result, when one calculates the matrix elements of $S_{2}$ from Eq. (A6), one only needs to consider contributions to $\left\langle\left\langle\mathrm{i}^{\prime} \mathrm{f}^{\prime}, \mathrm{JM}_{\mathrm{J}}\left|\left\langle L_{1}(\mathrm{t}) L_{1}\left(\mathrm{t}^{\prime}\right)\right\rangle\right|\right.\right.$ if, $\left.\left.\mathrm{JM}_{\mathrm{J}}\right\rangle\right\rangle$ from anisotropic potentials and simply neglects the term of $\left\langle\left\langle\mathrm{i}^{\prime} \mathrm{f}^{\prime}, \mathrm{JM}_{\mathrm{J}}\left|\left\langle L_{1}(\mathrm{t})\right\rangle\left\langle L_{1}\left(\mathrm{t}^{\prime}\right)\right\rangle\right|\right.\right.$ if, JM $\left.\left.\mathrm{J}\right\rangle\right\rangle$. Given the fact that the isotropic potentials can be treated as ordinal functions and matrix elements associated with them are always diagonal in the line space, the above claim should also be applicable here. For simplicity, we do not introduce new 
notations for the anisotropic part of potentials, rather than remind the readers to keep in mind that when one calculates the matrix elements of $\mathrm{S}_{2}$, the potentials mean their anisotropic parts only.

\section{Matrices of $S_{2, \text { outer,i }}, S_{2, \text { outer,f }}$, and $S_{2, \text { middle }}$ in the coordinate representation}

As further steps, one needs to evaluate the matrix elements of $S_{2 \text {,outer,i, }} S_{2, \text { outer, }}$, and $S_{2 \text {,middle }}$ based on anisotropic potentials. The research history on this subject shows that one could encounter convergence problems when the short-range site-site potential models are considered. In these cases, to adopt higher cut-offs in the spherical expansion of potentials and to consider all contributions including couplings between terms in the same tensor categories requires very much tedious work and numerical evaluations of a large number of resonance functions so that one may not be able to obtain converged results. As a result, to obtain converged results could become a formidable obstacle in practical calculations of these matrix elements.

In order to completely overcome the convergence problems, one can introduce the coordinate representation by choosing the orientations of the pair of molecules as the basis set in Hilbert space; i.e., $\left|\delta\left(\Omega_{a}-\Omega_{a \alpha}\right)\right\rangle \otimes\left|\delta\left(\Omega_{b}-\Omega_{b \alpha}\right)\right\rangle$ where $\Omega_{\mathrm{a} \alpha}$ and $\Omega_{\mathrm{b} \alpha}$ represent orientations of the absorber and bath molecules specified by $\alpha$, respectively. ${ }^{16,23}$ In contrast, with the standard representation, the basis set is constructed from $\left|i m_{i}\right\rangle \otimes\left|i_{2} m_{2}\right\rangle$, the product of the states of two interacting molecules. The advantage in choosing the coordinate representation results from a fact that with this representation, the potential becomes a diagonal operator and its matrix elements become multi-dimensional integrations whose angular parts can be evaluated analytically.

With the coordinate representation, one is able to write the matrix elements of $S_{2, \text { outer, }}$ as

$$
\begin{aligned}
S_{2, \text { outer }, i}^{i^{\prime} f^{\prime}, \text { if }}\left(r_{c}\right)= & \frac{1}{\hbar^{2}} \int_{-\infty}^{\infty} d t \int_{-\infty}^{t} d t^{\prime}\left\langle\left\langle i^{\prime} f^{\prime}, J M_{J}\left|\left\langle\mathcal{L}_{1}(t) \mathcal{L}_{1}\left(t^{\prime}\right)\right\rangle\right| i f, J M_{J}\right\rangle\right\rangle_{\text {outer }, i} \\
= & \frac{\delta_{j_{i}^{\prime} j_{i}} \delta_{f^{\prime} f}}{\hbar^{2}\left(2 j_{i}+1\right)} \int_{-\infty}^{\infty} d t \int_{-\infty}^{t} d t^{\prime} \sum_{i_{2} m_{2}} \rho_{i_{2}} \sum_{m_{i}} \sum_{i^{\prime \prime} m_{i}^{\prime \prime}} \sum_{i_{2}^{\prime} m_{2}^{\prime}} e^{i\left(\omega_{i^{\prime} i^{\prime \prime}}+\omega_{i_{2} i_{2}^{\prime}}\right) t} e^{-i\left(\omega_{i i^{\prime \prime}}+\omega_{i_{2} i_{2}}\right) t^{\prime}} \\
& \times \int d \Omega_{\alpha} \int d \Omega_{\beta}\left\langle i^{\prime} m_{i} i_{2} m_{2} \mid \alpha\right\rangle V_{\alpha}(R(t))\left\langle\alpha \mid i^{\prime \prime} m_{i}^{\prime \prime} i_{2}^{\prime} m_{2}^{\prime}\right\rangle\left\langle i^{\prime \prime} m_{i}^{\prime \prime} i_{2}^{\prime} m_{2}^{\prime} \mid \beta\right\rangle V_{\beta}\left(R\left(t^{\prime}\right)\right)\left\langle\beta \mid i m_{i} i_{2} m_{2}\right\rangle
\end{aligned}
$$

where $\quad \omega_{i i^{\prime \prime}}=\left[E^{(a)}(i)-E^{(a)}\left(i^{\prime \prime}\right)\right] / \hbar, \quad \omega_{i_{2} i_{2}^{\prime}}=\left[E^{(b)}\left(i_{2}\right)\right.$ $\left.-E^{(b)}\left(i_{2}^{\prime}\right)\right] / \hbar$, and $|\alpha\rangle$ is a shorthand notation for the basis set in the coordinate representation of the molecular pair and the subscript $\alpha$ of $\mathrm{V}_{\alpha}$ represents the potential evaluated at a specified orientation labeled by $\alpha$. The inner products $\left\langle\mathrm{i} \quad \mathrm{m}_{\mathrm{i}} \quad \mathrm{i}_{2} \mathrm{~m}_{2} \mid \alpha\right\rangle$ represent a transformation between two basis sets of these two representations and are nothing but products of the complex conjugates of the absorber molecular wave functions at the orientation $\Omega_{\mathrm{a} \alpha}$ and the complex conjugates of the bath molecular wave functions at the orientation $\Omega_{\mathrm{b} \alpha}$. Similarly, one is able to obtain the

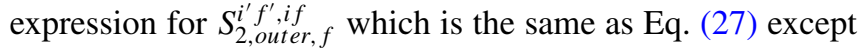
for making exchanges between the quantum numbers $\mathrm{i}$ and $\mathrm{f}^{\prime}$, between $\mathrm{i}^{\prime}$ and $\mathrm{f}$, and making switches between $\mathrm{t}$ and $\mathrm{t}^{\prime}$. Meanwhile with Eq. (26), one can obtain an expression for $S_{2, \text { middle }}^{i^{\prime} f^{\prime}, \text { as }}$

$$
\begin{aligned}
S_{2, \text { middle }}^{i^{\prime} f^{\prime}, i f}\left(r_{c}\right)= & \frac{1}{\hbar^{2}} \int_{-\infty}^{\infty} d t \int_{-\infty}^{t} d t^{\prime}\left\langle\left\langle i^{\prime} f^{\prime}, J M_{J}\left|\left\langle\mathcal{L}_{1}(t) \mathcal{L}_{1}\left(t^{\prime}\right)\right\rangle\right| i f, J M_{J}\right\rangle\right\rangle_{\text {middle }} \\
= & -\frac{\hbar^{-2}}{2 J+1} \int_{-\infty}^{\infty} d t \int_{-\infty}^{\infty} d t^{\prime} \sum_{i_{2} m_{2}} \rho_{i_{2}} \sum_{i_{2}^{\prime} m_{2}^{\prime}} \sum_{(m)} e^{i\left(\omega_{i^{\prime} i}+\omega_{i_{2} i_{2}}\right) t} e^{i\left(\omega_{f f^{\prime}}+\omega_{i_{2} i_{2}^{\prime}}\right) t^{\prime}} \\
& \times(-1)^{j_{f}-m_{f}+j_{f}^{\prime}-m_{f}^{\prime}} C\left(i^{\prime} f^{\prime} J, m_{i}^{\prime}-m_{f}^{\prime} M_{J}\right) C\left(i f J, m_{i}-m_{f} M_{J}\right) \\
& \times \int d \Omega_{\alpha} \int d \Omega_{\beta}\left\langle i^{\prime} m_{i}^{\prime} i_{2}^{\prime} m_{2}^{\prime} \mid \alpha\right\rangle V_{\alpha}(R(t))\left\langle\alpha \mid i m_{i} i_{2} m_{2}\right\rangle\left\langle f m_{f} i_{2} m_{2} \mid \beta\right\rangle V_{\beta}\left(R\left(t^{\prime}\right)\right)\left\langle\beta \mid f^{\prime} m_{f}^{\prime} i_{2}^{\prime} m_{2}^{\prime}\right\rangle .
\end{aligned}
$$

It is worth mentioning that as one considers the line mixing occurring among lines within the same bands, expressions for the matrix elements of $S_{2, \text { outer,i }}$ and $S_{2, \text { outer, }}$ can be simplified further. It is obvious that among lines within the same bands, both their initial and final vibrational quantum numbers are identical. This implies that one can replace $\delta_{j_{i}^{\prime} j_{i}}$ by $\delta_{i^{\prime} i}$ in the 
expression for $S_{2, \text { outer }, i}^{i^{\prime} f^{\prime}, i f}$ and replace $\delta_{j_{f} j_{f}^{\prime}}$ by $\delta_{f f^{\prime}}$ in $S_{2, \text { outer, } f}^{i^{\prime} f^{\prime}, \text { if }}$ As a result, the matrices of both these two terms are diagonal. But, this simplicity does not apply for the matrix of $S_{2 \text {,middle }}$ which remains as an off-diagonal matrix even within the same bands. With respect to this non diagonality of $\mathrm{S}_{2 \text {,middle, similar }}$ conclusions have been reported previously. $8,24,25$

For systems consisting of two linear molecules, formulas used to evaluate the diagonal matrix elements of the $S_{2, \text { outer, }}$,
$\mathrm{S}_{2, \text { outer,f }}$, and $\mathrm{S}_{2 \text {,middle }}$ terms are already available. ${ }^{16}$ Therefore, one only needs to derive a new expression for the off-diagonal

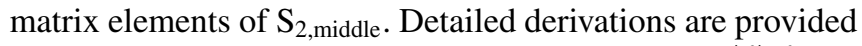
in Appendix $\mathrm{C}$ and the general expression for $S_{2, \text { middle }}^{i^{\prime} f^{\prime}, i f}$ is given by Eq. (C9).

For the $\mathrm{Q}$ lines of the $\mathrm{N}_{2}-\mathrm{N}_{2}$ pair where $\omega_{i^{\prime} i}=\omega_{f^{\prime} f}$, one is able to simplify Eq. (C9) further. By introducing the function $G_{L_{1} L_{2}}\left(t, t^{\prime}\right)$ defined in Ref. 16, Eq. (C5) can be expressed as

$$
\begin{aligned}
S_{2, \text { middle }}^{i^{\prime} f^{\prime}, i f}\left(r_{c}\right)= & (-1)^{j_{f}+j_{f}^{\prime}} \sqrt{\left(2 j_{i}^{\prime}+1\right)\left(2 j_{f}^{\prime}+1\right)\left(2 j_{i}+1\right)\left(2 j_{f}+1\right)} \\
& \times \sum_{L_{1} L_{2}}(-1)^{1+J+L_{1}} W\left(j_{i}^{\prime} j_{f}^{\prime} j_{i} j_{f}, J L_{1}\right) C\left(j_{i} j_{i}^{\prime} L_{1}, 000\right) C\left(j_{f}^{\prime} j_{f} L_{1}, 000\right) \\
& \times \sum_{i_{2} i_{2}^{\prime}}\left(2 i_{2}+1\right)\left(2 i_{2}^{\prime}+1\right) \rho_{i_{2}} C^{2}\left(i_{2} i_{2}^{\prime} L_{2}, 000\right) \int_{-\infty}^{\infty} d t \int_{-\infty}^{\infty} d t^{\prime} e^{i\left(\omega_{i^{\prime} i}+\omega_{i_{2} i_{2}}\right)\left(t-t^{\prime}\right)} G_{L_{1} L_{2}}\left(t, t^{\prime}\right) .
\end{aligned}
$$

Then, by manipulating the two dimensional integrations over $\mathrm{t}$ and $\mathrm{t}^{\prime}$, introducing the correlation functions $F_{L_{1} L_{2}}(t)$ and their Fourier transforms $H_{L_{1} L_{2}}(\omega)$ defined in Ref. 16, one can rewrite Eq. (29) as

$$
\begin{aligned}
S_{2, \text { middle }}^{i^{\prime} f^{\prime}, i f}\left(r_{c}\right)= & \sqrt{2 \pi}(-1)^{j_{f}+j_{f}^{\prime}} \sqrt{\left(2 j_{i}^{\prime}+1\right)\left(2 j_{f}^{\prime}+1\right)\left(2 j_{i}+1\right)\left(2 j_{f}+1\right)} \\
& \times \sum_{L_{1} L_{2}}\left\{(-1)^{1+J+L_{1}} W\left(j_{i}^{\prime} j_{f}^{\prime} j_{i} j_{f}, J L_{1}\right) C\left(j_{i} j_{i}^{\prime} L_{1}, 000\right) C\left(j_{f}^{\prime} j_{f} L_{1}, 000\right)\right\} \\
& \times \sum_{i_{2} i_{2}^{\prime}}\left(2 i_{2}+1\right)\left(2 i_{2}^{\prime}+1\right) \rho_{i_{2}} C^{2}\left(i_{2} i_{2}^{\prime} L_{2}, 000\right) H_{L_{1} L_{2}}\left(\omega_{i^{\prime} i}+\omega_{i_{2}^{\prime} i_{2}}\right) .
\end{aligned}
$$

We emphasize that Eq. (30) of $S_{2, \text { middle }}^{i^{\prime} f^{\prime}, \text { if }}$ is valid only for the Q lines. For other cases, i.e., infrared absorption lines in $\mathrm{P}$ and $\mathrm{R}$ branches, one has to use Eq. (C9) instead.

Because $S_{2 \text {,middle }}$ is the only term responsible for the off-diagonal matrix elements of $\mathrm{S}_{2}$, one can determine from Eq. (30) whether the line coupling could occur or not. In cases that the products of $\mathrm{C}\left(\mathrm{j}_{\mathrm{i}} \mathrm{j}_{\mathrm{i}}^{\prime} \mathrm{L}_{1}, 000\right) \mathrm{C}\left(\mathrm{j}_{\mathrm{f}}^{\prime} \mathrm{j}_{\mathrm{f}} \mathrm{L}_{1}, 000\right)$ are zero, there are no contributions to the line coupling between the lines of $\mathrm{f}^{\prime} \leftarrow \mathrm{i}^{\prime}$ and $\mathrm{f} \leftarrow \mathrm{i}$ through the Fourier transforms $H_{L_{1} L_{2}}(\omega)$ with the specified $L_{1}$. Therefore, the selection rules of these two Clebsch-Gordan coefficients play a crucial role in determining the line coupling. For the $\mathrm{N}_{2}-\mathrm{N}_{2}$ system, the tensor ranks $\mathrm{L}_{1}$ and $\mathrm{L}_{2}$ of the Fourier transforms $H_{L_{1} L_{2}}(\omega)$ must be even. ${ }^{16}$ As a result, the line coupling only occurs for those pairs of lines both whose initial quantum numbers (i.e., $\mathrm{j}_{\mathrm{i}}$ and $\mathrm{j}_{\mathrm{i}}^{\prime}$ ) and whose final quantum numbers (i.e., $\mathrm{j}_{\mathrm{f}}$ and $\mathrm{j}_{\mathrm{f}}^{\prime}$ ) have the same evenness or oddness. In addition, because functions of $H_{L_{1} L_{2}}(\omega)$ with lower $\mathrm{L}_{1}$ are usually more important than those with higher $L_{1}$, the closer between $j_{i}$ and $j_{i}^{\prime}$ and between $\mathrm{j}_{\mathrm{f}}$ and $\mathrm{j}_{\mathrm{f}}^{\prime}$ are, the stronger the line coupling is.

\section{Matrices of $-S_{2}$ and $\exp \left(-S_{2}\right)$ for Raman $Q$ lines of the $\mathrm{N}_{2}-\mathrm{N}_{2}$ pair}

In the present study, because the $S_{1}$ term is zero, the operator of $-i S_{1}-S_{2}$ can be replaced by $-S_{2}$. In addition, the imaginary parts of $S_{2, \text { outer, }}$ and $S_{2 \text {,outer, }}$ cancel out exactly for $\mathrm{Q}$ lines in the rigid rotor limit. ${ }^{18}$ Then, because $\mathrm{S}_{2 \text {,middle }}$ is real, the whole matrix of $-\mathrm{S}_{2}$ becomes a real one. As explained above, its off-diagonal matrix elements solely come from contributions from $S_{2 \text {,middle }}$. As a result, it is the $S_{2 \text {,middle }}$ terms that plays a crucial role to determine whether the isolated line approximation is applicable or not here.

Before carrying out numerical calculations, we can draw several conclusions from Eq. (30). If one chooses 4 as the cut-off for $\mathrm{L}_{1}$ and $\mathrm{L}_{2}$, there are six Fourier transforms $H_{L_{1} L_{2}}(\omega)$ associated with different values of $\mathrm{L}_{1}$ and $\mathrm{L}_{2}$. Among these functions, $H_{22}(\omega)$ contains contributions from the leading quadrupole-quadrupole interaction between two $\mathrm{N}_{2}$ molecules and thus, is the most important one. In practical calculations, instead of $\omega$ one uses a dimensionless parameter $k \equiv \omega\left(r_{c} / \bar{v}\right)$ where $\bar{v}$ is the mean velocity. In order to match results reported in Ref. 18 , our numerical calculations are carried out at $\mathrm{T}=298 \mathrm{~K}$ and at this temperature, the mean velocity is around $6.712 \times 10^{4} \mathrm{~cm} / \mathrm{s}$. We note that because $H_{22}(\omega)$ is associated with specified trajectories, it also depends on the parameter $r_{c}$. In Fig. 1, we present the profile of $H_{22}\left(k, r_{c}\right)$ as a two-dimensional function of $\mathrm{k}$ and $\mathrm{r}_{\mathrm{c}}$. As shown in the figure, its larger magnitudes occur only at a small $\mathrm{r}_{\mathrm{c}}$ region. Although the magnitudes of $\mathrm{H}_{22}\left(k, r_{c}\right)$ decrease as $\mathrm{k}$ increases, but roughly speaking, they still remain noticeable until at least $\mathrm{k}$ is beyond 14 or so. On the other hand, if $\omega$ is 


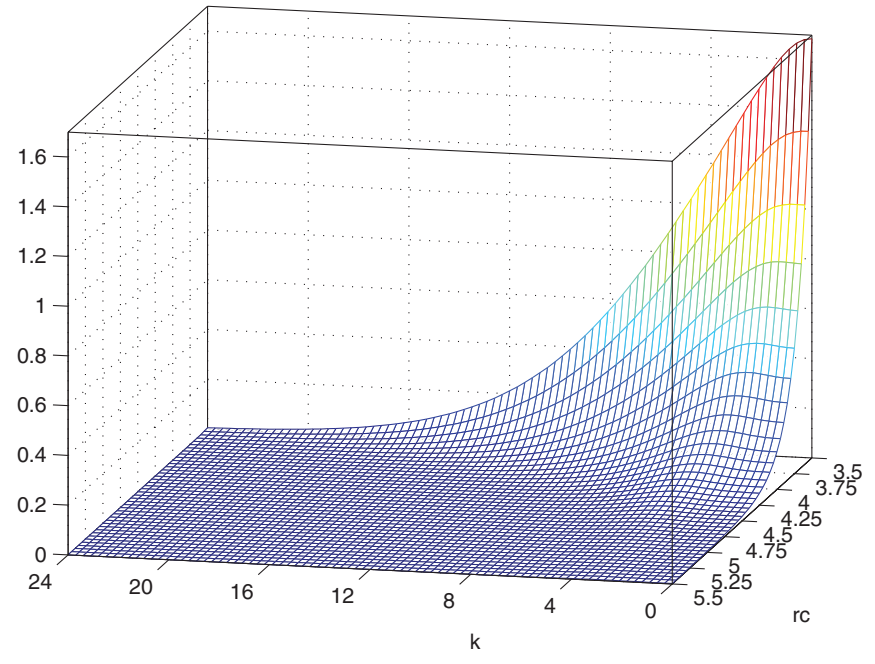

FIG. 1. Fourier transform of $H_{22}\left(k, r_{c}\right)\left(\right.$ in $\left.\mathrm{ps}^{-2}\right)$ at $\mathrm{T}=298 \mathrm{~K}$ for the $\mathrm{N}_{2}-\mathrm{N}_{2}$ pair as a two dimensional function of $\mathrm{k}$ (dimensionless) and $\mathrm{r}_{\mathrm{c}}$ (in $\AA$ ).

given in $\mathrm{cm}^{-1}$, its corresponding $\mathrm{k}$ value is about $0.1 \omega$. This implies that the Fourier transform of $H_{L_{1} L_{2}}\left(\omega_{i^{\prime} i}+\omega_{i_{2}^{\prime} i_{2}}\right)$ in Eq. (30) could make noticeable contributions to the offdiagonal matrix of $S_{2}$ unless its arguments of $\omega_{i^{\prime} i}+\omega_{i_{2}^{\prime} i_{2}}$ are beyond $140 \mathrm{~cm}^{-1}$ or so. Given the fact that the rotational constant of $\mathrm{N}_{2}$ is only about $2 \mathrm{~cm}^{-1}$ and values of $\omega_{i_{2}^{\prime} i_{2}}$ could be as small as zero, there is no reason to claim the line coupling can be ignored. Because $\mathrm{j}$ values of two coupled lines must at least differ by 2 , the minimum of their initial energy gaps is $4(2 \mathrm{j}+3) \mathrm{cm}^{-1}$. Based on these numbers, it is obvious that there are many coupled lines whose energy gaps are less than $140 \mathrm{~cm}^{-1}$. As a result, the off-diagonal matrix elements of $-\mathrm{S}_{2}$ are not negligible at all, and one must consider the line coupling for the Raman $Q$ lines of the $\mathrm{N}_{2}-\mathrm{N}_{2}$ pair.
For the Raman Q transitions, one can simply label lines by a number of $Q(j)\left(=j_{i}=j_{f}\right)$. As explained above, the line coupling occurs only among lines with the same evenness or the oddness of $Q(j)$. As a result, the matrix of $-S_{2}$ in the line space consists of two sub-matrices. One is constructed by lines with even $Q(j)$ values and another with odd $Q(j)$ values. In our numerical calculations, we have set 31 as the maximum of $Q(j)$. Then, both these two sub-matrices are $16 \times 16$ matrices whose sizes are very reasonable to deal with. In the present study, we have adopted the "exact" trajectory model. In order to cover all important trajectories well, we have selected 700 points of $r_{c}$ with more dense points to depict nearly head-on collisions because the latter play a dominant role in calculating the half-width and shift. The potential model including its isotropic part used in our calculations is the same one $^{22}$ used by Thibault et al. in their recent work. ${ }^{18}$

In the following, we present some samples to show calculated matrices of $-\mathrm{S}_{2}$ and $\exp \left(-\mathrm{S}_{2}\right)$. According to our calculation, the minimum of the closest distance at $\mathrm{T}=298 \mathrm{~K}$ is $r_{c, \text { min }}=3.42664 \AA$. As a sample to represent nearly headon collisions, we select a matrix of $-S_{2}$ with $r_{c}=3.67734 \AA$. For explanation purpose, we only explicitly present a smaller sub-matrix of $-\mathrm{S}_{2}$ constructed by the first 8 coupled lines with $\mathrm{Q}(\mathrm{j})=0,2,4,6,8,10,12$, and 14. As shown in Matrix 1, this $8 \times 8$ matrix is real and asymmetric. In addition, in comparison with the diagonal matrix elements, the most near-by offdiagonal elements are comparable. This clearly demonstrates the isolated line approximation is not applicable here and the necessity to consider the line coupling. Finally, because we have set 4 as the cut-off for $L_{1}$ and $L_{2}$ in the calculations, the line coupling occurs only between lines whose $Q(j)$ values differ from each other by no more than 4 . If one chooses 2 as the cut-off, there is no coupling between lines whose $Q(j)$ differences are beyond 2 . In this case, the two sub-matrices will be tri-diagonal.

Matrix 1: A sub-matrix of $-S_{2}$ associated with even $Q(j)$.

$\left|\begin{array}{cccccccc}-14.1192 & 5.9477 & 0.3832 & 0 & 0 & 0 & 0 & 0 \\ 5.8249 & -10.3909 & 5.7690 & 0.2221 & 0 & 0 & 0 & 0 \\ 0.3648 & 5.5228 & -10.8162 & 5.5545 & 0.1302 & 0 & 0 & 0 \\ 0 & 0.1993 & 5.1939 & -9.9161 & 4.9012 & 0.0739 & 0 & 0 \\ 0 & 0 & 0.1064 & 4.4496 & -8.3002 & 4.0079 & 0.0396 & 0 \\ 0 & 0 & 0 & 0.0539 & 3.4982 & -6.5061 & 3.1225 & 0.0190 \\ 0 & 0 & 0 & 0 & 0.0259 & 2.5924 & -4.8986 & 2.3701 \\ 0 & 0 & 0 & 0 & 0 & 0.0114 & 1.8560 & -3.6083\end{array}\right|$.

If one applies the isolated line approximation in evaluating the operator $\exp \left(-\mathrm{S}_{2}\right)$, the matrix of $\exp \left(-\mathrm{S}_{2}\right)$ becomes diagonal. By adopting values of the exponential function whose arguments are the diagonal elements of $-S_{2}$ in Matrix 1, one can easily construct the matrix of $\exp \left(-\mathrm{S}_{2}\right)$ which is presented in Matrix 2. We note that it is this kind of diagonal matrices that is used in calculating half-widths and shifts with the RB formalism. 
Matrix 2: A sub-matrix of $\exp \left(-\mathrm{S}_{2}\right)$ with even $\mathrm{Q}(\mathrm{j})$ in the isolated line approximation.

\begin{tabular}{|cccccccc}
0.00000 & 0 & 0 & 0 & 0 & 0 & 0 & 0 \\
0 & 0.00003 & 0 & 0 & 0 & 0 & 0 & 0 \\
0 & 0 & 0.00002 & 0 & 0 & 0 & 0 & 0 \\
0 & 0 & 0 & 0.00005 & 0 & 0 & 0 & 0 \\
0 & 0 & 0 & 0 & 0.00025 & 0 & 0 & 0 \\
0 & 0 & 0 & 0 & 0 & 0.00149 & 0 & 0 \\
0 & 0 & 0 & 0 & 0 & 0 & 0.00746 & 0 \\
0 & 0 & 0 & 0 & 0 & 0 & 0 & 0.02710
\end{tabular} \mid.

Matrix 3: A sub-matrix of $\exp \left(-\mathrm{S}_{2}\right)$ associated with even $\mathrm{Q}(\mathrm{j})$.

$\left|\begin{array}{llllllll}0.02587 & 0.05518 & 0.06669 & 0.06758 & 0.05946 & 0.04442 & 0.02267 & 0.01151 \\ 0.05410 & 0.11592 & 0.14144 & 0.14546 & 0.13073 & 0.10056 & 0.06269 & 0.02821 \\ 0.06263 & 0.13547 & 0.16868 & 0.17899 & 0.16818 & 0.13738 & 0.09238 & 0.04504 \\ 0.05931 & 0.13020 & 0.16726 & 0.18612 & 0.18678 & 0.16632 & 0.12414 & 0.06728 \\ 0.04730 & 0.10606 & 0.14244 & 0.16929 & 0.18555 & 0.18464 & 0.15685 & 0.09644 \\ 0.03077 & 0.07104 & 0.10134 & 0.13131 & 0.16087 & 0.18326 & 0.18144 & 0.12916 \\ 0.01530 & 0.03670 & 0.05646 & 0.08124 & 0.11330 & 0.15046 & 0.17692 & 0.14879 \\ 0.00516 & 0.01291 & 0.02153 & 0.03444 & 0.05451 & 0.08383 & 0.11647 & 0.11680\end{array}\right|$.

Next, we present results obtained from the new method. Because the sub-matrix of $-S_{2}$ is a real asymmetric matrix, to construct the unitary transform required in Eq. (13) is not straightforward. There is a subroutine F10ECF available in NAG which enables one to directly calculate the matrix exponential $\exp \left(-\mathrm{S}_{2}\right)$. In Matrix 3, we present the sub-matrix of $\exp \left(-\mathrm{S}_{2}\right)$ where the matrix of $-\mathrm{S}_{2}$ is given in Matrix 1 . Matrix 3 provides true values of the matrix elements of $\exp \left(-\mathrm{S}_{2}\right)$. By comparing Matrix 2 and Matrix 3, it is obvious that these two matrices completely differ from each other. The big differences convincingly demonstrate the necessity to consider the line coupling in evaluating the matrix of $\exp \left(-S_{2}\right)$. Furthermore, the diagonal elements of Matrix 3 are significantly larger than the diagonal elements of Matrix 2. As shown in Eq. (12), these diagonal matrix elements appear as a factor of $1-\exp \left(-S_{2}\right)$ in the integrand of the half-width. Based on this argument, we are sure that due to ignoring the line coupling, the current RB formalism significantly overestimates the half-widths of these Raman Q lines. This prediction will be fully verified later.

So far, we only present samples of the $8 \times 8$ sub-matrices derived from a specified trajectory. In order to provide a whole picture, we go back to the results derived from $16 \times 16$ submatrices associated with $\mathrm{Q}(\mathrm{j})=0,2, \ldots, 30$. With excluding and including the line coupling, we have calculated the function of $1-\exp \left(-\mathrm{S}_{2}\left(\mathrm{r}_{\mathrm{c}}\right)\right)$ for each of the four selected $\mathrm{Q}$ lines with $\mathrm{Q}(\mathrm{j})=0,4,12$, and 20 and we present their profiles in Fig. 2. As shown in the figure, effects on these profiles from the line coupling are important. More explicitly, in regions with small $r_{c}$ values, magnitudes of $1-\exp \left(-S_{2}\left(r_{c}\right)\right)$ derived from excluding the line coupling are significantly larger than those including the line coupling. As $r_{c}$ increase, their gaps become narrower, and finally disappear. This implies that the line coupling mainly occurs for nearly head-on collisions and its effects become less important or even negligible for glancing collisions.

Because magnitudes of the factor $b\left(d b / d_{c}\right)$ appearing in the integrand of the half-width significantly increase as $r_{c}$ decreases, this factor would further enhance the gaps of $1-\exp \left(-\mathrm{S}_{2}\left(\mathrm{r}_{\mathrm{c}}\right)\right)$ shown in Fig. 2. In order to fully demonstrate whole effects on calculated half-widths from the line

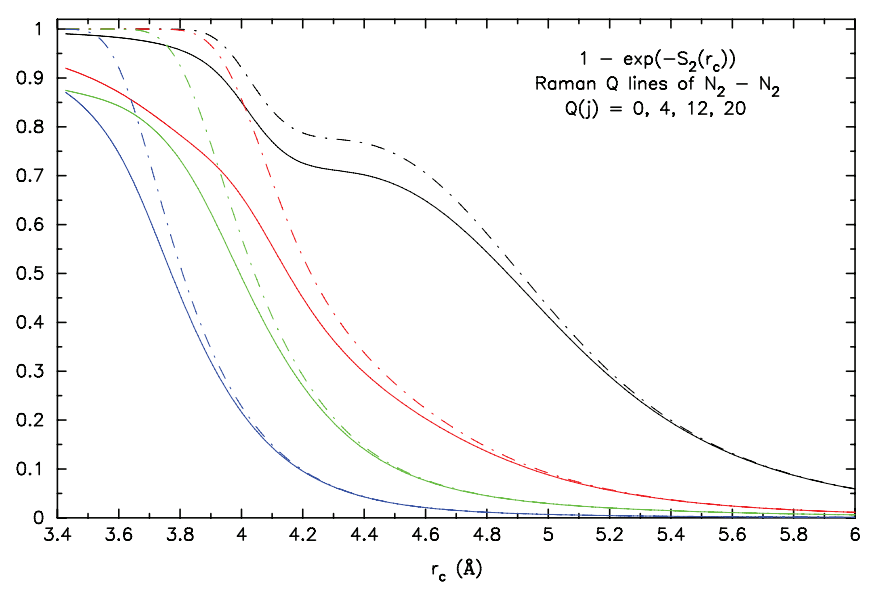

FIG. 2. Two sets of profiles of $1-\exp \left(-\mathrm{S}_{2}\left(\mathrm{r}_{\mathrm{c}}\right)\right)$ associated with four Raman $\mathrm{Q}$ lines of $\mathrm{N}_{2}-\mathrm{N}_{2}$ are plotted. They are obtained from the modified RB formalism without and with the line coupling and are plotted by dotted-dashed and solid lines, respectively. Different colors distinguish these Q lines with $\mathrm{Q}(\mathrm{j})=0$ (black), 4 (red), 12 (green), and 20 (blue). 


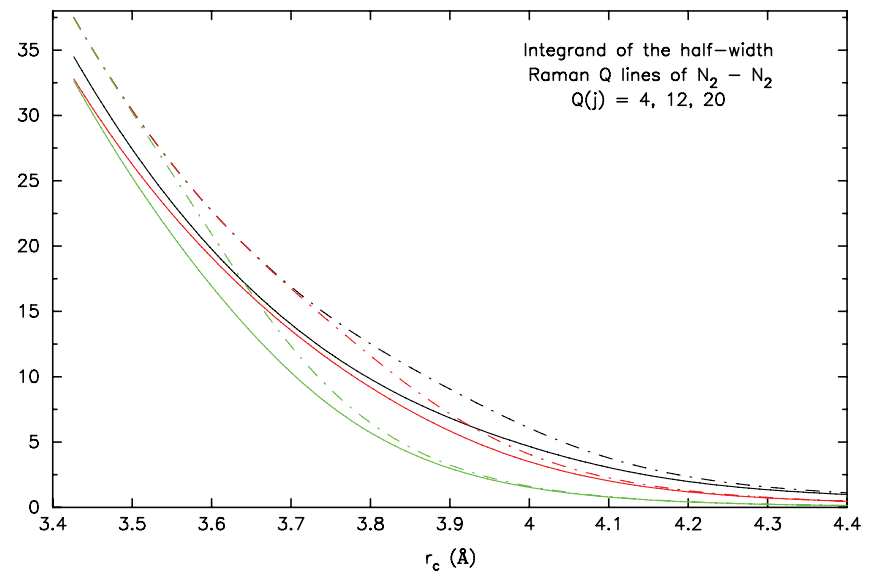

FIG. 3. The same as Fig. 2 except for two sets of profiles of the integrand $\mathrm{b}\left(\mathrm{db} / \mathrm{dr}_{\mathrm{c}}\right)\left[1-\exp \left(-\mathrm{S}_{2}\left(\mathrm{r}_{\mathrm{c}}\right)\right)\right]$ associated with three selected $\mathrm{Q}$ lines with $\mathrm{Q}(\mathrm{j})$ $=4$ (black), 12 (red), and 20 (green).

coupling, we present two sets of profiles of the integrand (i.e., $\left.b\left(d b / d r_{c}\right)\left[1-\exp \left(-S_{2}\left(r_{c}\right)\right)\right]\right)$ associated with the three $\mathrm{Q}$ lines with $\mathrm{Q}(\mathrm{j})=4,12$, and 20 in Fig. 3. As shown in the figure, magnitudes of the integrand without the line coupling, especially at a small $r_{c}$ region, are significantly larger than those derived from including the line coupling. Without any doubt, the plots indicate that by taking into account the line coupling, calculated half-widths would be significantly reduced.

\section{E. Matrix of the relaxation operator and calculated half-widths}

After all the matrix elements $\left\langle\left\langle i^{\prime} \mathrm{f}^{\prime}\left|\exp \left(-\mathrm{S}_{2}\left(\mathrm{r}_{\mathrm{c}}\right)\right)\right|\right.\right.$ if $\left.\rangle\right\rangle$ are available, it is easy to calculate not only the diagonal matrix elements of the relaxation operator $\langle\mathrm{m}(0)\rangle_{\text {bath }}$ denoted by W later but also its off-diagonal elements as well. Because the matrix elements of $\exp \left(-\mathrm{S}_{2}\left(\mathrm{r}_{\mathrm{c}}\right)\right)$ for the $\mathrm{N}_{2}-\mathrm{N}_{2}$ isotropic Raman $\mathrm{Q}$ lines are real, a simpler expression used to calculate the matrix elements of $\mathrm{W}$ can be expressed as

$$
\begin{aligned}
W^{i^{\prime} f^{\prime}, i f}= & \frac{n_{b} \bar{v}}{2 \pi c} \int_{r_{c, \text { min }}}^{+\infty} 2 \pi\left(b \frac{d b}{d r_{c}}\right) d r_{c} \\
& \times\left\{\delta_{i^{\prime} i} \delta_{f^{\prime} f}-\left\langle\left\langle i^{\prime} f^{\prime}\left|e^{-S_{2}\left(r_{c}\right)}\right| i f\right\rangle\right\rangle\right\} .
\end{aligned}
$$

By constructing two sub-spaces associated with even and odd $Q(j)$ lines and setting their up limits as $Q(j)=30$ and 31 , respectively, we have calculated two $16 \times 16$ sub-matrices of W. Here, we present the W matrix with even $Q(j)$ in Matrix 4. As is well known, ${ }^{5}$ the relaxation matrix should verify the detailed balance principle that connects upward and downward transitions and the so-called sum rule. For isotropic Raman Q lines, the sum rule claims that weighted sums of the matrix elements of $\mathrm{W}$ over columns are zero. The weighting factor of $\left[\left(2 j^{\prime} i+1\right) /\left(2 j_{i}+1\right)\right]^{1 / 2}$ is necessary introduced here because we have adopted Ben Reuven's conventions instead of Gordon's. ${ }^{26}$ All these points will be discussed in a forthcoming paper. In the present study, our main focus is to consider the diagonal elements of $\mathrm{W}$ because the latter are only the calculated half-widths.

Matrix 4: A $16 \times 16$ sub-matrix of the relaxation operator associated with even $Q(j)$.

$\left|\begin{array}{cccccccccccc}75.44 & -10.22 & -4.59 & -3.34 & -2.72 & -2.18 & -1.69 & -1.23 & -0.83 & \cdot & -0.00 & -0.00 \\ -10.05 & 61.80 & -12.22 & -7.78 & -6.20 & -4.96 & -3.83 & -2.80 & -1.89 & \cdot & -0.00 & -0.00 \\ -4.27 & -11.63 & 56.75 & -12.16 & -8.80 & -6.90 & -5.31 & -3.88 & -2.64 & \cdot & -0.01 & -0.00 \\ -2.92 & -6.89 & -11.13 & 53.94 & -12.77 & -8.99 & -6.77 & -4.95 & -3.39 & \cdot & -0.01 & -0.00 \\ -2.19 & -5.07 & -7.42 & -11.25 & 52.78 & -12.92 & -8.70 & -6.24 & -4.29 & \cdot & -0.01 & -0.00 \\ -1.59 & -3.66 & -5.24 & -7.13 & -10.90 & 51.77 & -12.83 & -8.23 & -5.57 & \cdot & -0.02 & -0.00 \\ -1.09 & -2.49 & -3.55 & -4.72 & -6.41 & -10.32 & 50.50 & -12.71 & -7.74 & \cdot & -0.04 & -0.01 \\ -0.68 & -1.57 & -2.23 & -2.96 & -3.94 & -5.60 & -9.72 & 48.76 & -12.64 & \cdot & -0.07 & -0.01 \\ -0.39 & -0.89 & -1.28 & -1.70 & -2.27 & -3.16 & -4.84 & -9.22 & 46.44 & \cdot & -0.14 & -0.02 \\ \cdot & \cdot & \cdot & \cdot & \cdot & \cdot & \cdot & \cdot & \cdot & \cdot & \cdot & \cdot \\ -0.00 & -0.00 & -0.00 & -0.00 & -0.00 & -0.00 & -0.01 & -0.01 & -0.02 & \cdot & 22.60 & -9.46 \\ -0.00 & -0.00 & -0.00 & -0.00 & -0.00 & -0.00 & -0.00 & -0.00 & -0.00 & \cdot & -5.41 & 19.07\end{array}\right|$.

In Fig. 4, we present the new values of calculated halfwidths together with those derived from the modified RB formalism without the line coupling. For comparison, we also present results derived from the close coupling method ${ }^{18}$ and from two different measurements. ${ }^{27}$ As demonstrated by Thibault et al.'s recent work, ${ }^{18}$ calculated results both from the RB formalism and from the modified RB formalism overestimate the half-widths by large amounts. In contrast, by taking into account the line coupling, our new calculated val- ues are reduced by about $15 \%$. Thus, one can conclude that effects on calculated half-widths from the line coupling are important and our refinement of the RB formalism goes in the right direction. Of course, there are still large differences between the new results and measurements or values predicted by the close coupling method. This implies that after conquering the line coupling obstacle, it is necessary to continue efforts in refining and improving the RB formalism further. 


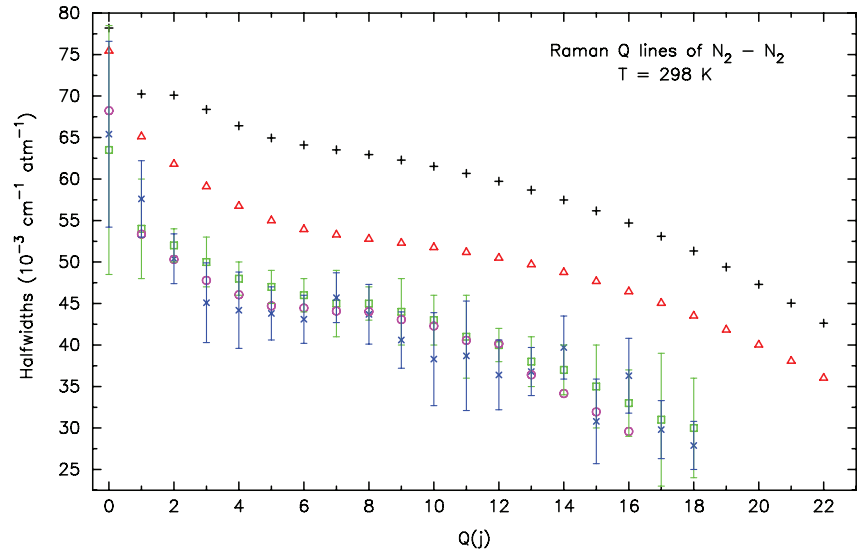

FIG. 4. Comparison of calculated half-widths and measurements for the Raman $Q$ lines of the $\mathrm{N}_{2}-\mathrm{N}_{2}$ pair. Theoretically calculated half-widths from the modified RB formalism without and with the line coupling are plotted by + and $\Delta$, respectively. Calculated values from the close coupling method ${ }^{18}$ are given by $\circ$. Meanwhile, two different measured results ${ }^{27}$ are plotted by $\square$ and $\times$, respectively.

\section{DISCUSSIONS AND CONCLUSIONS}

First of all, we would like to point out that one needs to distinguish the line mixing and the line coupling. Despite they share a common connection with applying the isolated line approximation, the line mixing and the line coupling describes different concepts. In the present study, the line mixing is associated with the approximation to deal with the resolvent operator $1 /\left(\omega-\mathrm{L}_{\mathrm{a}}-\langle\mathrm{m}(0)\rangle_{\text {bath }}\right)$ appearing in the spectral density $F(\omega)$ as shown in Eq. (5). Because the relaxation operator $\langle\mathrm{m}(0)\rangle_{\text {bath }}$ is off-diagonal in the line space, the denominator of the resolvent operator (i.e., $\omega$ $\left.-\mathrm{L}_{\mathrm{a}}-\langle\mathrm{m}(0)\rangle_{\text {bath }}\right)$ is off-diagonal too which causes difficulties in calculations. Meanwhile, the line coupling arises from how to calculate matrix elements of the Liouville scattering operator $\hat{\mathrm{S}}$ which is given in terms of an exponential form, no matter through the cumulant expansion in Eq. (9) or by some other way. ${ }^{19}$

Because the necessity to consider the line mixing and the line coupling have different criteria and the latter is more stringent than the former, in many situations the line mixing seldom occurs and the line coupling occurs very often or even almost always. As a result, to completely ignore the line coupling in developing line shape theories could cause more serious errors than that from ignoring the line mixing. In the present study, we focus our attention on effects on calculated half-widths from the line coupling only. However, as a subsequent outcome of the study shown in Matrix 4, the whole matrix elements of the relaxation operator are available. In a forthcoming study, we will investigate several topics related to these matrix elements, such as the detailed balance principle and the sum rules.

It is well known that to consider the line coupling is a longstanding problem in developing theories, and so far there are several methods proposed to overcome this challenge. Among them, the most important one is developed by Cherkasov through his continued contributions to this subject for more than three decades. Here, we briefly explain Cherkasov's formalism ${ }^{19}$ and outline differences between his method and ours. In developing his formalism, Cherkasov did not apply the cumulant expansion, but based on the usual perturbation expansions he directly put the scattering operator into the exponential form in an "ad hoc" way. Because the operators appearing in the exponential form depend on states of the bath molecule, he faced the same difficulties as that occurring in the RB formalism. In order to overcome these difficulties, he separated the relaxation operator into two parts: with respect to states of the bath molecule, one part is diagonal and another is off-diagonal. Then, by considering the second part as a perturbation and omitting the non-commutative feature between these two parts, he obtained an approximate expression for the relaxation operator in which only the first part appears in an exponential function and the second part appears linearly. As a result, one only needs to diagonalize smaller matrices associated with the first part whose dimension is only equal to the dimension of the line space of the absorber molecule. By comparing Cherkasov's formalism and our method, the latter's advantages are obvious. With our approach, one does not need to introduce the additional approximations to avoid his difficulties because any operations involving the bath molecule have been completely obviated. Besides, our method benefits from the cumulant expansion which has a sounder basis to deal with the perturbative expansion of the scattering operator.

The new method presented here is applicable for other linear molecular systems. There are many systems such as the $\mathrm{N}_{2}$ and $\mathrm{CO}$ mixtures, ${ }^{17} \mathrm{CO}_{2}-\mathrm{Ar}, \mathrm{C}_{2} \mathrm{H}_{2}-\mathrm{Ar}, \mathrm{CO}-\mathrm{Ar}, \mathrm{HCl}-\mathrm{Ar}$, $\mathrm{HF}-\mathrm{Ar}^{28}$ in which the $\mathrm{RB}$ formalism significantly overestimates the half-widths. Therefore, it is worth carrying out these calculations by applying the present method used here. Besides, it is worth to extend this method in considering effects from the line coupling on calculated shifts.

With respect to more complicated molecular systems involving symmetric-top and asymmetric top molecules, to apply the new method would not be straightforward. For the $\mathrm{H}_{2} \mathrm{O}-\mathrm{N}_{2}$ system, a lot of values of the half-width and shift listed in HITRAN come from theoretically calculated results with the RB formalism. For this important system, we expect that effects from the line coupling on calculated half-widths would not be small. Unfortunately, so far, there is lack of systematic investigations on this subject. Based on our considerations, for $\mathrm{H}_{2} \mathrm{O}$ lines in the same vibrational bands, the $\mathrm{S}_{2 \text {,middle }}$ term is still the only source responsible for the offdiagonal matrix elements of $S_{2}$. In this case, a new feature is that the leading correlation functions with $\mathrm{L}_{1}=1$ make major contributions to the off-diagonal elements. In contrast, it is well known that the correlation functions with odd $\mathrm{L}_{1}$ don't make any contributions to diagonal elements of $S_{2 \text {,middle }}$ at all. This indicates that one needs to carefully determine the coupling selection rules which are more complicated than that applicable for the $\mathrm{N}_{2}-\mathrm{N}_{2}$ system. Furthermore, the numbers of strong $\mathrm{H}_{2} \mathrm{O}$ lines in the same main bands are more likely beyond one thousand. As a result, to determine groups of coupled lines is not an easy job. But, thanks to the selection rules applying not only to the angular quantum number $\mathrm{j}$ but also to other indices $\mathrm{k}_{\mathrm{a}}$ and $\mathrm{k}_{\mathrm{c}}$, the numbers of lines in the same coupling groups would still remain reasonable. Meanwhile, for $\mathrm{H}_{2} \mathrm{O}$ lines belonging to different bands, the line coupling 
caused by intramolecular resonances (such as Coriolis, Fermi, Darling-Dennsion, and more complex resonances) could also occur. $^{29}$ In this case, besides the $S_{2 \text {,middle }}$ term, both $S_{2, \text { outer, }}$ and $S_{2, \text { outer, }}$ can make contributions to the off-diagonal matrix elements of $\mathrm{S}_{2}$.

Finally, as shown in Fig. 4, the new calculated halfwidths of the Raman $Q$ lines still do not match measurements or values obtained with the most accurate close coupling method. However, we do not consider the differences as bad signs for our current refinement effort. In our opinion, there are other weaknesses remaining in the RB formalism and among them, the most severe one is to treat the translational motion and the internal motion separately. More specifically, exchanges of their energies and angular momentums are not considered during collisional processes and collision trajectories are assumed to be independent of anisotropic potential models. It is understandable that one has to adopt this approximation because there are no alternatives available right now. But, we expect that these simplifications would bear a large uncertainty at least at the same level as that resulting from ignoring the line coupling. Besides, so far the third and higher orders of the perturbative expansion of the scattering operator have not been taken into account. Therefore, we consider the remaining gaps shown in Fig. 4 as room for further refinements.

\section{ACKNOWLEDGMENTS}

We thank the two reviewers for their helpful comments on the manuscript. Two of the authors (Q. Ma and R. H. Tipping) acknowledge financial support from National Science Foundation (NSF) under Grant No. 1228861. This research used resources of the National Energy Research Scientific Computing Center, which is supported by the Office of Science of the U.S. Department of Energy under Contract No. DE-AC02-05CH11231.

\section{APPENDIX A: THE CUMULANT EXPANSION}

It is well known that the cumulant expansion is a very useful tool in solving many physical problems. In order to apply this method in specified problems, an essential step is to define the so called average \langle\rangle accordingly. Besides the average must satisfy the normalization condition $\langle\mathrm{I}\rangle=1$, the choice of the average has considerable freedom. The average could be chosen as the diagonal matrix element of operators in a specified representation or something else. In addition, results of the average could be either ordinary numbers or quantum operators.

For the pressure broadening problem, one can apply the cumulant expansion in evaluating the matrix elements of the Liouville scattering operator $\hat{\mathrm{S}}$ whose perturbation expression is given by

$$
\begin{aligned}
\hat{S} & =1-\frac{i}{\hbar} \int_{-\infty}^{\infty} \mathcal{L}_{1}(t) d t-\frac{1}{\hbar^{2}} \int_{-\infty}^{+\infty} \mathcal{L}_{1}(t) d t \int_{-\infty}^{t} \mathcal{L}_{1}\left(t^{\prime}\right) d t^{\prime}+\cdots \\
& =\theta \exp \left(-\frac{i}{\hbar} \int_{-\infty}^{\infty} \mathcal{L}_{1}(t) d t\right)
\end{aligned}
$$

where $\mathcal{L}_{1}(\mathrm{t})$ is a Liouville operator associated with the Hilbert interaction operator $\hat{V}_{1}(t)\left(\equiv e^{i\left(H_{a}+H_{b}\right) t / \hbar} V e^{-i\left(H_{a}+H_{b}\right) t / \hbar}\right)$ and the symbol $\theta$ is the time ordering operator. By properly defining the average \langle\rangle , the average of $\hat{\mathrm{S}}$ can be expressed as

$$
\begin{aligned}
& \left\langle\theta \exp \left(-\frac{i}{\hbar} \int_{-\infty}^{\infty} \mathcal{L}_{1}(t) d t\right)\right\rangle \\
& =\exp \left\langle\theta \exp \left(-\frac{i}{\hbar} \int_{-\infty}^{\infty} \mathcal{L}_{1}(t) d t\right)-1\right\rangle_{c} \\
& =\exp \left\{-\frac{i}{\hbar} \int_{-\infty}^{\infty}\left\langle\mathcal{L}_{1}(t)\right\rangle_{c} d t\right. \\
& \left.-\frac{1}{\hbar^{2}} \int_{-\infty}^{+\infty} \int_{-\infty}^{t}\left\langle\mathcal{L}_{1}(t) \mathcal{L}_{1}\left(t^{\prime}\right)\right\rangle_{c} d t d t^{\prime}+\cdots\right\},
\end{aligned}
$$

where \langle\rangle$_{\mathrm{c}}$ is the so called cumulant average. A cumulant average is not the average defined, but it is related to the latter. For example, the first two cumulant averages in Eq. (A2) are given by

$$
\begin{aligned}
\left\langle\mathcal{L}_{1}(t)\right\rangle_{c} & =\left\langle\mathcal{L}_{1}(t)\right\rangle \\
\left\langle\mathcal{L}_{1}(t) \mathcal{L}_{1}\left(t^{\prime}\right)\right\rangle_{c} & =\left\langle\mathcal{L}_{1}(t) \mathcal{L}_{1}\left(t^{\prime}\right)\right\rangle-\left\langle\mathcal{L}_{1}(t)\right\rangle\left\langle\mathcal{L}_{1}\left(t^{\prime}\right)\right\rangle .
\end{aligned}
$$

Thus, up to the second order in Eq. (A2), the average of $\hat{S}$ can be written as

$$
\left\langle\theta \exp \left(-\frac{i}{\hbar} \int_{-\infty}^{\infty} \mathcal{L}_{1}(t) d t\right)\right\rangle=\exp \left(-i S_{1}-S_{2}\right)
$$

where $S_{1}$ and $S_{2}$ are defined by

$$
S_{1}=\frac{1}{\hbar} \int_{-\infty}^{\infty}\left\langle\mathcal{L}_{1}(t)\right\rangle d t
$$

and

$$
\begin{aligned}
S_{2}= & \frac{1}{\hbar^{2}}\left\{\int_{-\infty}^{+\infty} \int_{-\infty}^{t}\left\langle\mathcal{L}_{1}(t) \mathcal{L}_{1}\left(t^{\prime}\right)\right\rangle d t d t^{\prime}\right. \\
& \left.-\int_{-\infty}^{+\infty} \int_{-\infty}^{t}\left\langle\mathcal{L}_{1}(t)\right\rangle\left\langle\mathcal{L}_{1}\left(t^{\prime}\right)\right\rangle d t d t^{\prime}\right\},
\end{aligned}
$$

respectively.

\section{APPENDIX B: MATRIX ELEMENTS OF THE LIOUVILLE OPERATORS $L_{1}(\mathrm{t})$ AND $L_{1}(\mathrm{t}) L_{1}\left(\mathrm{t}^{\prime}\right)$ IN HILBERT SPACE}

In the following, we present derivations of the matrix elements of the Liouville operators $L_{1}(\mathrm{t})$ and $L_{1}(\mathrm{t}) L_{1}\left(\mathrm{t}^{\prime}\right)$ in Hilbert space. For the $\mathrm{N}_{2}-\mathrm{N}_{2}$ pair, the absorber states and the pertuber states are denoted by $\left|\mathrm{im}_{\mathrm{i}}\right\rangle$ and $\left|\mathrm{i}_{2} \mathrm{~m}_{2}\right\rangle$, respectively. In terms of the bases in Hilbert space, the matrix elements of the Liouville operator $L_{1}(\mathrm{t})$ can be written as

$$
\begin{gathered}
\left\langle\left\langle i^{\prime} m_{i}^{\prime} f^{\prime} m_{f}^{\prime}, i_{2}^{\prime} m_{2}^{\prime} i_{2}^{\prime} m_{2}^{\prime}\left|\mathcal{L}_{1}(t)\right| i m_{i} f m_{f}, i_{2} m_{2} i_{2} m_{2}\right\rangle\right\rangle \\
=\left\langle i^{\prime} m_{i}^{\prime} i_{2}^{\prime} m_{2}^{\prime}|\hat{V}(t)| i m_{i} i_{2} m_{2}\right\rangle \delta_{f^{\prime} m_{f}^{\prime}, f m_{f}} \delta_{i_{2} m_{2}, i_{2}^{\prime} m_{2}^{\prime}} \\
-\left\langle f m_{f} i_{2} m_{2}|\hat{V}(t)| f^{\prime} m_{f}^{\prime} i_{2}^{\prime} m_{2}^{\prime}\right\rangle \delta_{i m_{i}, i^{\prime} m_{i}^{\prime}} \delta_{i_{2}^{\prime} m_{2}^{\prime}, i_{2} m_{2}},
\end{gathered}
$$


where $\delta_{i m_{i}, i^{\prime} m_{i}^{\prime}}$ represents $\delta_{i i^{\prime}} \delta_{m_{i} m_{i}^{\prime}}$. Similarly, the matrix elements of $L_{1}(\mathrm{t}) L_{1}\left(\mathrm{t}^{\prime}\right)$ can be written as

$$
\begin{aligned}
\left\langle\left\langle i^{\prime} m_{i}^{\prime} f^{\prime} m_{f}^{\prime}, i_{2}^{\prime} m_{2}^{\prime} i_{2}^{\prime} m_{2}^{\prime}\left|\mathcal{L}_{1}(t) \mathcal{L}_{1}\left(t^{\prime}\right)\right| i m_{i} f m_{f}, i_{2} m_{2} i_{2} m_{2}\right\rangle\right\rangle \\
=\left\{\left\langle i^{\prime} m_{i}^{\prime} i_{2}^{\prime} m_{2}^{\prime}|\hat{V}(t)| i^{\prime \prime} m_{i}^{\prime \prime} i_{2}^{\prime \prime} m_{2}^{\prime \prime}\right\rangle \delta_{f^{\prime} m_{f}^{\prime}, f^{\prime \prime} m_{f}^{\prime \prime}} \delta_{i_{2}^{\prime \prime \prime} m_{2}^{\prime \prime \prime}, i_{2}^{\prime} m_{2}^{\prime}}\right. \\
\left.-\left\langle f^{\prime \prime} m_{f}^{\prime \prime} i_{2}^{\prime \prime \prime} m_{2}^{\prime \prime \prime}|\hat{V}(t)| f^{\prime} m_{f}^{\prime} i_{2}^{\prime} m_{2}^{\prime}\right\rangle \delta_{i^{\prime \prime} m_{i}^{\prime \prime}, i^{\prime} m_{i}^{\prime}} \delta_{i_{2}^{\prime} m_{2}^{\prime}, i_{2}^{\prime \prime} m_{2}^{\prime \prime}}\right\} \\
\times\left\{\left\langle i^{\prime \prime} m_{i}^{\prime \prime} i_{2}^{\prime \prime} m_{2}^{\prime \prime}\left|\hat{V}\left(t^{\prime}\right)\right| i m_{i} i_{2} m_{2}\right\rangle \delta_{f^{\prime \prime} m_{f}^{\prime \prime}, f m_{f}} \delta_{i_{2} m_{2}, i_{2}^{\prime \prime \prime} m_{2}^{\prime \prime \prime}}\right. \\
\left.-\left\langle f m_{f} i_{2} m_{2}\left|\hat{V}\left(t^{\prime}\right)\right| f^{\prime \prime} m_{f}^{\prime \prime} i_{2}^{\prime \prime \prime} m_{2}^{\prime \prime \prime}\right\rangle \delta_{i m_{i}, i^{\prime \prime} m_{i}^{\prime \prime}} \delta_{i_{2}^{\prime \prime} m_{2}^{\prime \prime}, i_{2} m_{2}}\right\} .
\end{aligned}
$$

After carrying out some algebraic works, one can rewrite Eq. (B2) as

$$
\begin{aligned}
\left\langle\left\langle i^{\prime} m_{i}^{\prime} f^{\prime} m_{f}^{\prime}, i_{2}^{\prime} m_{2}^{\prime} i_{2}^{\prime} m_{2}^{\prime}\left|\mathcal{L}_{1}(t) \mathcal{L}_{1}\left(t^{\prime}\right)\right| i m_{i} f m_{f}, i_{2} m_{2} i_{2} m_{2}\right\rangle\right\rangle \\
=\left\langle i^{\prime} m_{i}^{\prime} i_{2} m_{2}\left|\hat{V}(t) \hat{V}\left(t^{\prime}\right)\right| i m_{i} i_{2} m_{2}\right\rangle \delta_{f^{\prime} m_{f}^{\prime}, f m_{f}} \delta_{i_{2}^{\prime} m_{2}^{\prime}, i_{2} m_{2}} \\
\quad+\left\langle f m_{f} i_{2} m_{2}\left|\hat{V}\left(t^{\prime}\right) \hat{V}(t)\right| f^{\prime} m_{f}^{\prime} i_{2} m_{2}\right\rangle \delta_{i^{\prime} m_{i}^{\prime}, i m_{i}} \delta_{i_{2}^{\prime} m_{2}^{\prime}, i_{2} m_{2}} \\
\quad-\left\langle i^{\prime} m_{i}^{\prime} i_{2}^{\prime} m_{2}^{\prime}|\hat{V}(t)| i m_{i} i_{2} m_{2}\right\rangle\left\langle f m_{f} i_{2} m_{2}\left|\hat{V}\left(t^{\prime}\right)\right| f^{\prime} m_{f}^{\prime} i_{2}^{\prime} m_{2}^{\prime}\right\rangle \\
\quad-\left\langle f m_{f} i_{2} m_{2}|\hat{V}(t)| f^{\prime} m_{f}^{\prime} i_{2}^{\prime} m_{2}^{\prime}\right\rangle\left\langle i^{\prime} m_{i}^{\prime} i_{2}^{\prime} m_{2}^{\prime}\left|\hat{V}\left(t^{\prime}\right)\right| i m_{i} i_{2} m_{2}\right\rangle .
\end{aligned}
$$

As shown by Eq. (B3), the matrix elements of $L_{1}(\mathrm{t}) L_{1}\left(\mathrm{t}^{\prime}\right)$ consist of four terms.

\section{APPENDIX C: EXPRESSION FOR THE MATRIX ELEMENTS OF $\mathbf{S}_{2, \text { middle }}$}

Starting from the general expression for $S_{2, \text { middle }}^{i^{\prime} f^{\prime}, \text { gif }}$ given by Eq. (28), we present detailed derivations here. First of all, based on the general expression for the interaction, ${ }^{16}$

$$
\begin{aligned}
V(\vec{R}(t))= & \sum_{L_{1} L_{2} L} u\left(L_{1} L_{2} L ; R(t)\right) \sum_{m_{1} m_{2} m} C\left(L_{1} L_{2} L, m_{1} m_{2} m\right) \\
& \times Y_{L_{1} M_{1}}\left(\Omega_{a}\right) Y_{L_{2} M_{2}}\left(\Omega_{b}\right) Y_{L m}^{*}(\omega(t)) .
\end{aligned}
$$

The integration over $\Omega_{\alpha}$ in Eq. (28) is given by

$$
\begin{aligned}
\int & d \Omega_{\alpha}\left\langle i^{\prime} m_{i}^{\prime} i_{2}^{\prime} m_{2}^{\prime} \mid \alpha\right\rangle V_{\alpha}(R(t))\left\langle\alpha \mid i m_{i} i_{2} m_{2}\right\rangle \\
= & \sum_{L_{1} L_{2} L} \sum_{\mu_{1} \mu_{2} M} u\left(L_{1} L_{2} L, R(t)\right) C\left(L_{1} L_{2} L, \mu_{1} \mu_{2} M\right) \\
& \times\left\{\int d \Omega_{a \alpha} Y_{j_{i}^{\prime} m_{i}^{\prime}}^{*}\left(\Omega_{a \alpha}\right) Y_{j_{i} m_{i}}\left(\Omega_{a \alpha}\right) Y_{L_{1} \mu_{1}}\left(\Omega_{a \alpha}\right)\right\} \\
& \times\left\{\int d \Omega_{b \alpha} Y_{i_{2}^{\prime} m_{2}^{\prime}}^{*}\left(\Omega_{b \alpha}\right) Y_{i_{2} m_{2}}\left(\Omega_{b \alpha}\right) Y_{L_{2} \mu_{2}}\left(\Omega_{b \alpha}\right)\right\} Y_{L M}^{*}\left(\omega_{\alpha}(t)\right) .
\end{aligned}
$$

Then, by analytically carrying out the integrations over $\Omega_{\mathrm{a} \alpha}$ and $\Omega_{\mathrm{b} \alpha}$, Eq. (C2) can be written as

$$
\begin{aligned}
\int & d \Omega_{\alpha}\left\langle i^{\prime} m_{i}^{\prime} i_{2}^{\prime} m_{2}^{\prime} \mid \alpha\right\rangle V_{\alpha}(R(t))\left\langle\alpha \mid i m_{i} i_{2} m_{2}\right\rangle \\
= & \frac{1}{4 \pi} \sum_{L_{1} L_{2} L} \sqrt{\frac{\left(2 j_{i}^{\prime}+1\right)\left(2 j_{i}+1\right)\left(2 i_{2}^{\prime}+1\right)\left(2 i_{2}+1\right)}{\left(2 L_{1}+1\right)\left(2 L_{2}+1\right)}} u\left(L_{1} L_{2} L, R(t)\right) \\
& \times C\left(j_{i} j_{i}^{\prime} L_{1}, 000\right) C\left(i_{2} i_{2}^{\prime} L_{2}, 000\right) \sum_{\mu_{1} \mu_{2} M}(-1)^{m_{i}+m_{2}} C\left(L_{1} L_{2} L, \mu_{1} \mu_{2} M\right) \\
& \times C\left(j_{i} j_{i}^{\prime} L_{1},-m_{i} m_{i}^{\prime} \mu_{1}\right) C\left(i_{2} i_{2}^{\prime} L_{2},-m_{2} m_{2}^{\prime} \mu_{2}\right) Y_{L M}^{*}\left(\omega_{\alpha}(t)\right) .
\end{aligned}
$$

With respect to the integration over $\Omega_{\beta}$ in Eq. (28), one can obtain a result similar to Eq. (C3). Then, one can rewrite Eq. (28) as

$$
\begin{aligned}
S_{2, \text { middle }}^{i^{\prime} f^{\prime}, i f}\left(r_{c}\right)=- & \frac{\hbar^{-2}}{16 \pi^{2}(2 J+1)} \sum_{i_{2} m_{2}} \rho_{i_{2}} \sum_{i_{2}^{\prime} m_{2}^{\prime}} \sum_{(m)}\left(2 i_{2}+1\right)\left(2 i_{2}^{\prime}+1\right) \\
& \times \sum_{L_{1} L_{2} L} \sum_{L_{1}^{\prime} L_{2}^{\prime} L^{\prime}} \sqrt{\frac{\left(2 j_{i}^{\prime}+1\right)\left(2 j_{f}^{\prime}+1\right)\left(2 j_{i}+1\right)\left(2 j_{f}+1\right)}{\left(2 L_{1}+1\right)\left(2 L_{2}+1\right)\left(2 L_{1}^{\prime}+1\right)\left(2 L_{2}^{\prime}+1\right)}} \\
& \times C\left(j_{i} j_{i}^{\prime} L_{1}, 000\right) C\left(j_{f}^{\prime} j_{f} L_{1}^{\prime}, 000\right) C\left(i_{2} i_{2}^{\prime} L_{2}, 000\right) C\left(i_{2}^{\prime} i_{2} L_{2}^{\prime}, 000\right) \\
& \times \int_{-\infty}^{\infty} d t \int_{-\infty}^{\infty} d t^{\prime} e^{i\left(\omega_{i^{\prime} i}+w_{i_{2} i_{2}}\right) t} e^{i\left(\omega_{f f^{\prime}}+w_{i_{2} i_{2}^{\prime}}\right) t^{\prime}} u\left(L_{1} L_{2} L, R(t)\right) u\left(L_{1}^{\prime} L_{2}^{\prime} L^{\prime}, R\left(t^{\prime}\right)\right) \\
& \times(-1)^{j_{f}+j_{f}^{\prime}+m_{i}-m_{f}} C\left(j_{i}^{\prime} j_{f}^{\prime} J, m_{i}^{\prime}-m_{f}^{\prime} M_{J}\right) C\left(j_{i} j_{f} J, m_{i}-m_{f} M_{J}\right) \\
& \times \sum_{\mu_{1} \mu_{2} M} \sum_{\mu_{1}^{\prime} \mu_{2}^{\prime} M^{\prime}}(-1)^{m_{2}+m_{2}^{\prime}} C\left(L_{1} L_{2} L, \mu_{1} \mu_{2} M\right) C\left(L_{1}^{\prime} L_{2}^{\prime} L^{\prime}, \mu_{1}^{\prime} \mu_{2}^{\prime} M^{\prime}\right) \\
& \times C\left(j_{i} j_{i}^{\prime} L_{1},-m_{i} m_{i}^{\prime} \mu_{1}\right) C\left(j_{f}^{\prime} j_{f} L_{1}^{\prime},-m_{f}^{\prime} m_{f} \mu_{1}^{\prime}\right) C\left(i_{2} i_{2}^{\prime} L_{2},-m_{2} m_{2}^{\prime} \mu_{2}\right) \\
& \times C\left(i_{2}^{\prime} i_{2} L_{2}^{\prime},-m_{2}^{\prime} m_{2} \mu_{2}^{\prime}\right) Y_{L M}^{*}\left(\omega_{\alpha}(t)\right) Y_{L^{\prime} M^{\prime}}^{*}\left(\omega_{\beta}\left(t^{\prime}\right)\right) .
\end{aligned}
$$


In Eq. (C4), there are thirteen magnetic quantum numbers of which only five are independent. By carrying out summations over four independent ones, the expression of Eq. (C4) can be simplified as

$$
\begin{aligned}
S_{2, \text { middle }}^{i^{\prime} f^{\prime}, i f}\left(r_{c}\right)= & (-1)^{j_{f}+j_{f}^{\prime}} \sqrt{\left(2 j_{i}^{\prime}+1\right)\left(2 j_{f}^{\prime}+1\right)\left(2 j_{i}+1\right)\left(2 j_{f}+1\right)} \\
& \times \sum_{L_{1} L_{2}}(-1)^{1+J+L_{1}} W\left(j_{i}^{\prime} j_{f}^{\prime} j_{i} j_{f}, J L_{1}\right) C\left(j_{i} j_{i}^{\prime} L_{1}, 000\right) C\left(j_{f}^{\prime} j_{f} L_{1}, 000\right) \\
& \times \sum_{i_{2} i_{2}^{\prime}}\left(2 i_{2}+1\right)\left(2 i_{2}^{\prime}+1\right) \rho_{i_{2}} C^{2}\left(i_{2} i_{2}^{\prime} L_{2}, 000\right) \frac{\hbar^{-2}}{16 \pi^{2}\left(2 L_{1}+1\right)\left(2 L_{2}+1\right)} \\
& \times \sum_{L}(-1)^{L_{1}+L_{2}+L} \sum_{M} \int_{-\infty}^{\infty} d t \int_{-\infty}^{\infty} d t^{\prime} e^{i\left(\omega_{i^{\prime} i}+\omega_{i_{2} i_{2}}\right) t} e^{i\left(\omega_{f f^{\prime}}+\omega_{i_{2} i_{2}^{\prime}}\right) t^{\prime}} \\
& \times u\left(L_{1} L_{2} L, R(t)\right) u\left(L_{1} L_{2} L, R\left(t^{\prime}\right)\right) Y_{L M}^{*}\left(\omega_{\alpha}(t)\right) Y_{L M}\left(\omega_{\beta}\left(t^{\prime}\right)\right) .
\end{aligned}
$$

Because in general $\omega_{\mathrm{i}^{\prime} \mathrm{i}} \neq \omega_{\mathrm{f}^{\prime} \mathrm{f}}$, our previous method ${ }^{16}$ used in deriving the diagonal matrix elements of $S_{2, \text { outer, },}, S_{2, \text { outer, } f}$, and $\mathrm{S}_{2, \text { middle }}$ is not applicable here and one has to separately carry out the integrations over $\mathrm{t}$ and $\mathrm{t}^{\prime}$ in Eq. (C5). First of all, we rewrite the integrations as

$$
\begin{aligned}
& \sum_{M}(-1)^{M} \int_{-\infty}^{\infty} d t e^{i\left(\omega_{i^{\prime} i}+\omega_{i_{2}^{\prime} i_{2}}\right) t} u\left(L_{1} L_{2} L, R(t)\right) Y_{L-M}(\omega(t)) \\
& \quad \times \int_{-\infty}^{\infty} d t^{\prime} e^{i\left(\omega_{f f^{\prime}}+\omega_{i_{2} i_{2}^{\prime}}\right) t^{\prime}} u\left(L_{1} L_{2} L, R\left(t^{\prime}\right)\right) Y_{L-M}\left(\omega\left(t^{\prime}\right)\right) .
\end{aligned}
$$

(C6)
Then, in terms of the Fourier transforms $\Phi_{L_{1} L_{2} L M}(\omega)$ defined by

$$
\Phi_{L_{1} L_{2} L M}(\omega)=\frac{1}{\sqrt{2 \pi}} \int_{-\infty}^{\infty} d t e^{i \omega t} u\left(L_{1} L_{2} L, R(t)\right) Y_{L M}(\omega(t)),
$$

the first integration in Eq. (C6) can be expressed as

$$
\begin{aligned}
& \int_{-\infty}^{\infty} d t e^{i\left(\omega_{i^{\prime} i}+\omega_{i_{2}^{\prime} i_{2}}\right) t} u\left(L_{1} L_{2} L, R(t)\right) Y_{L-M}(\omega(t)) \\
& =\sqrt{2 \pi} \Phi_{L_{1} L_{2} L-M}\left(\omega_{i^{\prime} i}+\omega_{i_{2}^{\prime} i_{2}}\right)
\end{aligned}
$$

Similarly, the second integration is given by $\sqrt{2 \pi} \Phi_{L_{1} L_{2} L M}\left(\omega_{f f^{\prime}}+\omega_{i_{2} i_{2}^{\prime}}\right)$. Finally, the expression for the off-diagonal matrix elements of $S_{2 \text {,middle }}$ can be written as

$$
\begin{aligned}
S_{2, \text { middle }}^{i^{\prime} f^{\prime}, i f}\left(r_{c}\right)= & (-1)^{j_{f}+j_{f}^{\prime}} \sqrt{\left(2 j_{i}^{\prime}+1\right)\left(2 j_{f}^{\prime}+1\right)\left(2 j_{i}+1\right)\left(2 j_{f}+1\right)} \\
& \times \sum_{L_{1} L_{2}}(-1)^{1+J+L_{1}} W\left(j_{i}^{\prime} j_{f}^{\prime} j_{i} j_{f}, J L_{1}\right) C\left(j_{i} j_{i}^{\prime} L_{1}, 000\right) C\left(j_{f}^{\prime} j_{f} L_{1}, 000\right) \\
& \times \sum_{i_{2} i_{2}^{\prime}}\left(2 i_{2}+1\right)\left(2 i_{2}^{\prime}+1\right) \rho_{i_{2}} C^{2}\left(i_{2} i_{2}^{\prime} L_{2}, 000\right) \frac{1}{8 \pi \hbar^{2}\left(2 L_{1}+1\right)\left(2 L_{2}+1\right)} \\
& \times \sum_{L}(-1)^{L_{1}+L_{2}+L} \sum_{M}(-1)^{M} \Phi_{L_{1} L_{2} L-M}\left(\omega_{i^{\prime} i}+\omega_{i_{2}^{\prime} i_{2}}\right) \Phi_{L_{1} L_{2} L M}\left(\omega_{f f^{\prime}}+\omega_{i_{2} i_{2}^{\prime}}\right)
\end{aligned}
$$

${ }^{1}$ R. G. Gordon, J. Chem. Phys. 44, 3083 (1966); 45, 1649 (1966).

${ }^{2}$ P. W. Anderson, Phys. Rev. 76, 647 (1949).

${ }^{3}$ C. J. Tsao and B. Curnutte, J. Quant. Spectrosc. Radiat. Transf. 2, 41 (1962).

${ }^{4}$ S. Green, J. Chem. Phys. 62, 2271 (1975).

${ }^{5}$ J. M. Hartmann, C. Boulet, and D. Robert, Collisional Effects on Molecular Spectra (Elsevier, 2008)

${ }^{6}$ J. Buldyreva, N. N. Laventieva, and V. I. Starikov, Collisional Line Broadening and Shifting at Atmospheric Gases: A Practical Guile for Line Shape Modelling by Current Semi-Classical Approaches (Imperial College Press, 2011).
${ }^{7}$ R. H. Tipping and R. M. Herman, J. Quant. Spectrosc. Radiat. Transf. 10, 881 (1970).

${ }^{8}$ J. Bonamy, L. Bonamy, and D. Robert, J. Chem. Phys. 67, 4441 (1977).

${ }^{9}$ C. Bloch, Nucl. Phys. 7, 451 (1958).

${ }^{10}$ R. P. Leavitt and D. Korff, J. Chem. Phys. 74, 2180 (1981).

${ }^{11}$ D. Robert and J. Bonamy, J. Phys. 40, 923 (1979).

${ }^{12}$ A. D. Bykov, N. N. Lavrentieva, and L. N. Sinitsa, Atmos. Oceanic Opt. 5, 587 (1992); 5, 728 (1992).

${ }^{13}$ J. Buldyreva, J. Bonamy, and D. Robert, J. Quant. Spectrosc. Radiat. Transf. 62, 321 (1999). 
${ }^{14}$ Q. Ma, R. H. Tipping, and C. Boulet, J. Quant. Spectrosc. Radiat. Transf. 103, 588 (2007).

${ }^{15}$ R. Kubo, J. Phys. Soc. Jpn. 17, 1100 (1962).

${ }^{16}$ Q. Ma, R. H. Tipping, and C. Boulet, J. Chem. Phys. 124, 014109 (2006).

${ }^{17}$ M. Afzelius, P.-E. Bengtsson, and J. Bonamy, J. Chem. Phys. 120, 8616 (2004).

${ }^{18}$ F. Thibault, L. Gomez, S. V. Ivanov, O. G. Buzykin, and C. Boulet, J. Quant. Spectrosc. Radiat. Transf. 113, 1887 (2012) and references therein.

${ }^{19}$ M. R. Cherkasov, Opt. Spectrosc. 40, 3 (1976); 105, 851 (2008); 106, 1 (2009); 107, 553 (2009).

${ }^{20}$ U. Fano, Phys. Rev. 131, 259 (1963).

${ }^{21}$ A. Ben-Reuven, Phys. Rev. 141, 34 (1966); 145, 7 (1966).
${ }^{22}$ D. Cappelletti, F. Pirani, B. Bussery-Honvault, L. Gomez, and M. Bartolomei, Phys. Chem. Chem. Phys. 10, 4281 (2008)

${ }^{23}$ Q. Ma and R. H. Tipping, J. Chem. Phys. 108, 3386 (1998); 111, 5909 (1999); 112, 574 (2000); 116, 4102 (2002).

${ }^{24} \mathrm{G}$. Buffa and O. Tarrini, Phys. Rev. A 16, 1612 (1977).

${ }^{25}$ G. Buffa, A. Di Lieto, O. Minguzzi, O. Tarrini, and M. Tonelli, Phys. Rev. A 34, 1065 (1986).

${ }^{26}$ R. Shafer and R. G. Gordon, J. Chem. Phys. 58, 5422 (1973).

${ }^{27}$ F. Thibault, R. Z. Martínez, D. Bermejo, and L. Gómez, J. Quant. Spectrosc. Radiat. Transf. 112, 2542 (2011).

${ }^{28}$ S. V. Ivanov and O. G. Buzykin, J. Quant. Spectrosc. Radiat. Transf. 119, 84 (2013).

${ }^{29}$ A. D. Bykov, N. N. Lavrentieva, L. N. Sinitsa, and A. M. Solodov, Atmos. Oceanic Opt. 14, 774 (2001) 